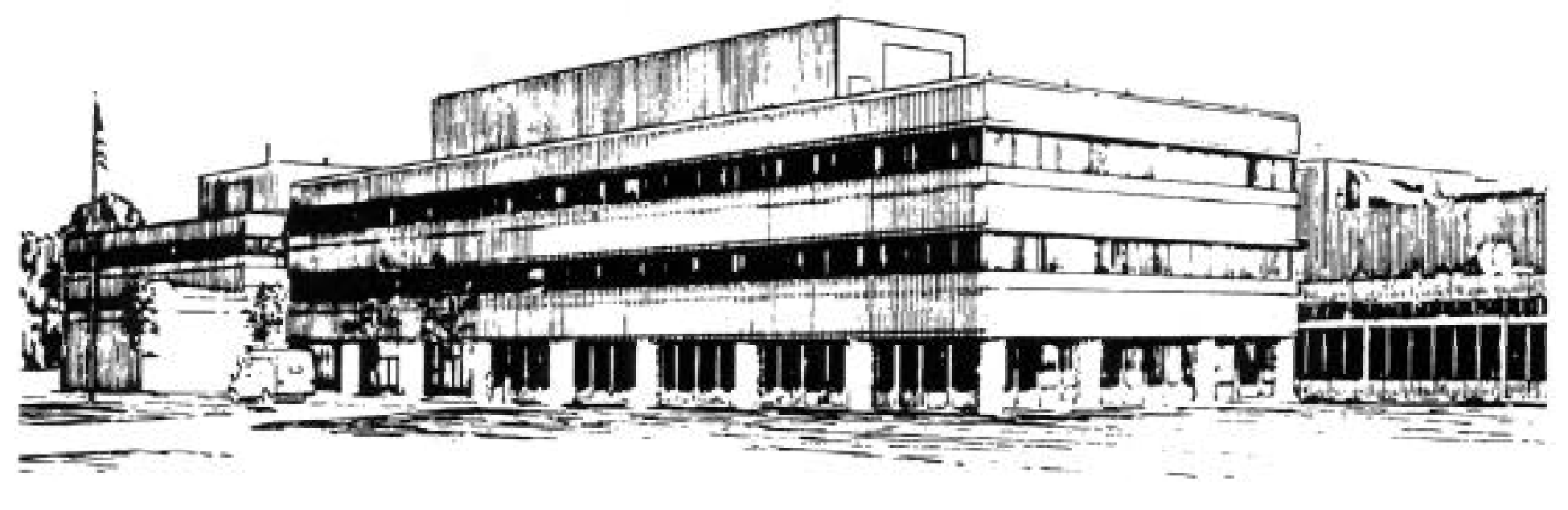




\section{PPPL Reports Disclaimer}

This report was prepared as an account of work sponsored by an agency of the United States Government. Neither the United States Government nor any agency thereof, nor any of their employees, makes any warranty, express or implied, or assumes any legal liability or responsibility for the accuracy, completeness, or usefulness of any information, apparatus, product, or process disclosed, or represents that its use would not infringe privately owned rights. Reference herein to any specific commercial product, process, or service by trade name, trademark, manufacturer, or otherwise, does not necessarily constitute or imply its endorsement, recommendation, or favoring by the United States Government or any agency thereof. The views and opinions of authors expressed herein do not necessarily state or reflect those of the United States Government or any agency thereof.

\section{Availability}

This report is posted on the U.S. Department of Energy's Princeton Plasma Physics Laboratory Publications and Reports web site in Calendar Year 2000. The home page for PPPL Reports and Publications is: http://www.pppl.gov/pub_report/

DOE and DOE Contractors can obtain copies of this report from:

U.S. Department of Energy

Office of Scientific and Technical Information

DOE Technical Information Services (DTIS)

P.O. Box 62

Oak Ridge, TN 37831

Telephone: (865) 576-8401

Fax: (865) 576-5728

Email: reports@adonis.osti.gov

This report is available to the general public from:

National Technical Information Service

U.S. Department of Commerce

5285 Port Royal Road

Springfield, VA 22161

Telephone: $1-800-553-6847$ or

(703) $605-6000$

Fax: (703) 321-8547

Internet: http://www.ntis.gov/ordering.htm 


\title{
Cosmic Ray Pitch Angle Scattering Through $90^{\circ}$
}

\author{
G.M. Felice ${ }^{1}$ and R.M. Kulsrud ${ }^{2}$ \\ Princeton Plasma Physics Laboratory \\ Princeton University \\ New Jersey, 08542 USA
}

Received —

\footnotetext{
${ }^{1}$ new address: Max-Planck-Institut für Kernphysik - Heidelberg, Germany

${ }^{2}$ Peyton Hall, Princeton University, Princeton, ND 08542 USA
} 


\begin{abstract}
We study the problem of cosmic ray diffusion in the galactic disk with particular attention to the problem of particle scattering through the $\theta=\cos ^{-1}\left(v_{\|} / v\right)=$ $90^{\circ}$ pitch angle in momentum space by wave-particle mirror interaction (here $v_{\|}$ is the cosmic ray velocity parallel to the average galactic magnetic field). We consider the case in which cosmic rays are the only source of magnetic turbulence, which originates as the relativistic particles try to stream through the interstellar plasma faster than the local Alfven speed. The wave growth rate is proportional to the cosmic ray anisotropy and the amplitude of hydro-magnetic waves generated by this streaming instability is limited by the presence of various damping mechanisms. We study the propagation of cosmic rays in the different phases of the I.S.M., in particular the coronal regions (where the main form of wave dissipation is non-linear Landau damping) and the warm regions (where charge exchange between the ions and the neutral atoms gives rise to the dominant form of wave dissipation). We also account for ion-cyclotron damping of small wavelength waves. The effect of a spectrum of waves is to limit the anisotropy of the cosmic ray distribution function, and hence to limit their drift velocity. We show that quasi-linear resonant scattering cannot account for particle diffusion everywhere in momentum space, and that particles with $\theta \sim 90^{\circ}$ must change their pitch angle by mirror interaction with long wavelength waves generated by the $\theta \sim 0$ particles. We match the quasi-linear scattering with the adiabatic mirroring in a small boundary layer in momentum space close to the $\theta \sim 90^{\circ}$ point and then we calculate the diffusion coefficient together with the cosmic ray drift velocity. We show that scattering through the $90^{\circ}$ point is very efficient and we calculate the correction to the particle diffusion coefficient due to the presence of mirroring.
\end{abstract}


Subject headings: Cosmic Rays, $90^{\circ}$ pitch angle

\section{Introduction}

Cosmic rays strongly interact with the galactic magnetic field and their diffusion in space is regulated by this interaction. According to quasi-linear theory, cosmic rays scatter off waves of wavelength comparable to their gyroradius, giving rise to an effective diffusion in pitch angle which, in turn, regulates their real space diffusion (Kulsrud and Pearce 1969; Wentzel 1969). The waves are predominantly low frequency hydro-magnetic waves. Although their origin could be due to the presence of some source of galactic magnetic turbulence, driven by gas turbulence or stellar activity it is more likely that cosmic rays themselves, as they try to stream faster then the local Alfven speed through a magnetized plasma, generate them by the resonant streaming instability. In this paper we focus our attention to this second possibility. Although quasi-linear theory has proven successful in explaining the physics of the propagation of cosmic rays, the question of scattering through a pitch angle of $90^{\circ}$ has not yet been successfully resolved. The $90^{\circ}$ problem has been analyzed by several authors in the course of the years, in particular Völk (1973), Klimas and Sandri (1973) and Goldstein $(1976,1980)$. In this paper we wish to present a self-consistent, yet simple theory, that resolves the problem by including mirror interaction with waves self generated by quasi-linear interaction itself.

Throughout this paper we make use of the result that the waves propagate mainly parallel to the magnetic field and only waves propagating in one direction are excited. Thus $k_{\perp} \rho \ll 1$ (where $\rho$ is the perpendicular particle gyroradius) and $k_{\|} \approx k$ and we can work in the frame of reference moving with the waves. The validity of this result is discussed in Appendix B. The waves propagate in the same direction as the particle drift velocity. In this frame the waves are static, i.e. $\omega_{r}=0$ and the energy of the cosmic rays remain 
constant.

In this frame the wave-particle resonant condition is (Kulsrud and Pearce 1969):

$$
k_{r}=\frac{1}{\mu r_{L}}
$$

where $r_{L}=v / \Omega$ is the proton Larmor radius, $\Omega$ is the relativistic gyro-frequency and $\mu=\cos (\theta)=v_{\|} / v$ where $\theta$ is the particle pitch angle. Eq. (1) shows that cosmic rays of larger pitch angle $(\mu \rightarrow 0)$ interact with waves of shorter wavelength. The $90^{\circ}$ problem arises from the fact that there is very little wave energy in small wavelength waves. Hence, particles of large pitch angle are unable to scatter across the $\theta=90^{\circ}$ point by resonant scattering. If no other process can account for their crossing of $90^{\circ}$, a large anisotropy would appear in the cosmic ray distribution function together with a drift velocity comparable to the speed of light, contrary to observations.

In this paper we consider a model in which the source of cosmic rays and their sink are connected by a magnetic flux tube of length $L_{z}$ and study particle diffusion parallel to the magnetic field as they propagate through the interstellar medium. We first study the propagation of particles in the coronal regions of the disk, where the typical ion temperature is $T_{i} \approx 10^{6} \mathrm{~K}$ and the average ion density is $n_{i} \approx 10^{-3} \mathrm{~cm}^{-3}$. Then we discuss propagation in the warm regions, where $T_{i} \approx 10^{4} \mathrm{~K}$ and $n_{i} \approx 0.03 \mathrm{~cm}^{-3}$, where there is a large fraction of neutral particles, with density $n_{H} \approx 0.2 \mathrm{~cm}^{-3}$, which linearly damp the waves. Throughout this paper we will take a value of $B_{o} \approx 3 \mu \mathrm{G}$ for the galactic magnetic field strength.

We show that the $90^{\circ}$ problem is resolved once we include the effect of mirroring of particles of $\theta \sim 90^{\circ}$ by MHD waves quasilinearly generated by the $\theta \sim 0$ resonant particles. Although this mirror and the quasilinear wave interaction act over all pitch angles, the mirror interaction dominates for $\theta$ very near $90^{\circ}$, while the quasilinear interaction dominates 
outside of this small region. Therefore we treat the problem as a boundary layer problem. By solving the cosmic ray diffusion equation and the wave spectrum equation in steady state we calculate self-consistently the cosmic ray distribution function and the wave spectrum everywhere in pitch angle space. We show that the distribution function is well behaved at $\theta=90^{\circ}$ (see Fig. 5 where we plot the normalized distribution function with and without the effect of mirroring). We then determine the particle diffusion coefficient and show that its expression is given by the familiar one (Cesarsky 1980) plus a correction due to the presence of the mirror region which appears as the natural logarithm of $\mu_{c}=\cos \left(\theta_{c}\right)$, where $\theta_{c}$ is the critical value of the pitch angle above which mirror interaction becomes dominant. The value of $\theta_{c}$ is determined self-consistently from the problem.

The paper is organized as follows. In Sections 2 and 3 we restrict ourselves to cosmic rays of a few $\mathrm{GeV}$. In Sec. 2 we determine the wave spectrum and the cosmic ray distribution function self-consistently in the quasilinear region by solving the steady state cosmic ray kinetic equation and by balancing the growth rate and the linear or non-linear damping rate. In Sec. 3 we include the effect of the mirror interaction and solve for the wave spectrum and cosmic ray distribution function in the boundary layer near $\theta=90^{\circ}$. We numerically solve the kinetic equation for the cosmic ray distribution function to match onto the quasilinear region. Finally, we calculate the cosmic ray diffusion coefficient and drift velocity. In Sec. 4 we expend our analysis to include cosmic rays of many $\mathrm{GeV}$ and derive the energy dependence of the cosmic ray drift velocity. In Sec. 5 we draw our conclusions and indicate open problems and possibilities for future research. 


\section{Quasi-Linear Scattering of Cosmic Ray Protons of a few GeV}

\subsection{The Diffusion Equation}

We first consider cosmic ray protons of a few $\mathrm{GeV}$. Thus, we set $\gamma \approx 5$ and neglect relativistic effects. The kinetic equation for the cosmic ray distribution function $F(p, \mu, z, t)$ is (Kulsrud and Pearce 1969):

$$
\frac{\partial F}{\partial t}+\dot{\mu} \frac{\partial F}{\partial \mu}+v_{z} \frac{\partial F}{\partial z}=\frac{\partial}{\partial \mu}\left[\frac{1-\mu^{2}}{2} \nu(\mu) \frac{\partial F}{\partial \mu}\right]+S(p, z)
$$

where the term proportional to $\dot{\mu}$ represents the mirror interaction. In the quasi-linear region considered in this section we drop this term. $S(p, z)$ is the source (or sink) of cosmic rays and $\nu(\mu)$ is the wave-particle collision frequency defined in terms of the wave spectrum $I(\mathbf{k})$ as:

$$
\nu(\mu)=\frac{\pi}{4} \Omega_{o}^{2} \int d^{3} k I(\mathbf{k}) \delta\left(k v_{z}-\Omega_{o}\right)
$$

Here $I(\mathbf{k})$ is the energy spectrum of MHD waves normalized to $B_{o}^{2} / 8 \pi$, where $B_{o}$ is the

background magnetic field and $\Omega_{o}=\Omega(\gamma=5)$ is the non-relativistic gyrofrequency. The delta function $\delta\left(k_{z} v_{z}-\Omega_{o}\right)$ represents the resonant condition. Note that only the first harmonic wave-particle interaction is present, since higher harmonic interaction is absent in the $k_{\perp}=0$ limit. The resonant particles are then the particles whose pitch angle satisfies the resonant condition, Eq. (1), with $r_{L}=r_{o}=c / \Omega_{o}$. By performing the integration over the delta function we obtain:

$$
\nu(\mu)=\frac{\pi}{4} \Omega_{o} \mathcal{E}_{r}(\mu)
$$

where $\mathcal{E}=\left(\delta B / B_{o}\right)^{2}$ and $\mathcal{E}_{r}$ represents the energy in the waves at the resonant wavenumber 
$k_{r}$ :

$$
\mathcal{E}_{r}(\mu)=k_{r} I\left(k_{r}\right)=\frac{1}{\mu r_{o}} \int d^{2} k_{\perp} I\left(\frac{1}{\mu r_{o}}, k_{\perp}\right)
$$

To simplify the notation we will omit the subscript "r". We solve Eq. (2) in the limit of strong scattering by writing $F=F_{o}+F_{1}+F_{2}+\ldots$, with $F_{1} / F_{o} \ll 1$ and $F_{2} / F_{1} \ll 1$. To lowest order the distribution function is isotropic, i.e. $F_{0}=F_{0}(p, z, t)$. Integrating Eq. (2) to first order (assuming quasi-steady state and weak source) we have:

$$
\frac{\partial F_{1}}{\partial \mu}=-\frac{v}{\nu(\mu)} \frac{\partial F_{0}}{\partial z}=\frac{4}{\pi} \frac{r_{o}}{L_{z}} \frac{1}{\mathcal{E}(\mu)}
$$

valid in the quasi-linear region, i.e. for $|\mu|>\mu_{c}$. In deriving Eq. (6), we have introduced the normalized $\mathcal{F}=F_{1} / F_{0}$ and $L_{z}$ is the cosmic ray density scale length, $L_{z}^{-1} \equiv-\partial \ln \left(F_{o}\right) / \partial z$. The flux of cosmic rays in the $\hat{z}$ direction (along $B_{o}$ ) is:

$$
\Phi_{z}=2 \pi v \int F_{1} \mu d \mu p^{2} d p=2 \pi v \int_{-1}^{1} \mathcal{F} \mu d \mu \int_{p_{o}}^{\infty} F_{o} p^{2} d p=\frac{n_{c . r .}^{\text {tot }} v}{2} \int_{-1}^{1} \mathcal{F} \mu d \mu
$$

Defining the bulk drift velocity of the cosmic rays by $V_{d r i f t}=\Phi_{z} / n_{c . r \text {. }}^{\text {tot }}$ we have:

$$
\frac{V_{\text {drift }}}{v}=\frac{1}{2} \int_{-1}^{1} \mathcal{F} \mu d \mu
$$

We can obtain $\mathcal{F}$ from Eq. (6) in the quasilinear region $\left(|\mu|>\mu_{c}\right)$ once we know the wave spectrum. To find the contribution to the drift velocity coming from the $|\mu|<\mu_{c}$ region we need to include the mirror interaction and solve the boundary layer problem, and that will be done in the next section. 


\subsection{The Growth Rate}

In the limit of $k_{\perp} \rho \ll 1$ and $\gamma \approx 1$ the expression for the growth rate of hydro-magnetic waves valid in the reference frame of the waves is (Kulsrud and Pearce 1969):

$$
\Gamma_{g}(k)=\pi^{2} e^{2}\left(\frac{V_{A}}{c}\right)^{2} \int d^{3} p v_{\perp}^{2} \frac{1}{V_{A} p} \frac{\partial F(p, \mu)}{\partial \mu} \delta\left(k v_{z}-\Omega_{o}\right)
$$

Taking a power law for $F_{o}, F_{o} \propto p^{-n}$, we have:

$$
\Gamma(\mu)=\Omega_{o}\left(\frac{n-3}{n-2}\right) \frac{\pi}{8}\left(\frac{c}{V_{A}}\right)\left(\frac{n_{c . r .}^{t o t}}{n_{i}}\right) \mu\left[1-\mu^{2}\right]\left[\frac{\partial \mathcal{F}(\mu)}{\partial \mu}\right]
$$

where $n_{c . r .}^{t o t}$ is the total number of cosmic rays. Throughout this paper we will use the experimental value of $n=4.7$ when evaluating expressions.

Eq. (10) shows that the growth rate is proportional to the anisotropy $\delta \simeq \mathcal{F}$ of the cosmic ray distribution function. In turn, as we see from Eq. (6), the anisotropy is inversely proportional to the quasi-linear scattering rate, so that it is also inversely proportional to the energy in the waves. Then the picture arises in which the cosmic ray anisotropy acts to create a spectrum of waves, which in turn act back on the anisotropy to reduce it. We combine Eq. (6) and Eq. (10) to obtain:

$$
\Gamma(\mu)=\left(\frac{n-3}{n-2}\right) \frac{\Omega_{o}}{2}\left(\frac{c}{V_{A}}\right)\left(\frac{n_{c . r .}^{\text {tot }}}{n_{i}}\right)\left(\frac{r_{o}}{L_{z}}\right)\left(1-\mu^{2}\right) \frac{\mu}{\mathcal{E}(\mu)} .
$$

We now determine the wave spectrum by balancing the growth rate and the damping rate assuming that the waves are marginally stable. 


\subsection{The Wave Spectrum in the Coronal Regions of the I.S.M.}

A large portion of the galactic disk is filled with a low density, hot plasma immersed in the galactic large scale magnetic field which is of the order of few microgauss (Cowie and Songaila 1986; Spitzer 1978). This phase of the interstellar gas is known as the coronal phase, its typical parameters being an average ion density of $10^{-3} \mathrm{~cm}^{-3}$ and a temperature

of the order of $10^{6} \mathrm{~K}$. Parallel propagating MHD waves do not suffer linear damping. Of the non-linear damping mechanisms possible, the most important is non-linear Landau damping (Kulsrud 1978; Völk and Cesarsky 1982), which arises when two circularly polarized Alfven waves beat together to produce a third pseudo wave that has a variation in the magnitude of the field strength. The beat wave exerts a force on the background thermal ions, and when the ion thermal distribution is Maxwellian, energy flows from the wave with higher frequency (larger k-vector) to the wave with the lower frequency and to the thermal plasma ions. Hence high frequency waves are damped by the presence of low frequency waves and by the presence of thermal particles. We write the unsaturated damping rate as (Kulsrud 1978):

$$
\Gamma_{n . l .}=\frac{1}{2} \sqrt{\frac{\pi}{2}}\left[\frac{<v_{\perp}^{4}>}{8 V_{i}^{4}}\right] V_{i} k \mathcal{E}(k)
$$

where the average $\left\langle v_{\perp}^{4}\right\rangle$ is to be taken over particles which are not trapped in the beat wave. For a thermal distribution in which no particles are trapped we simply have $<v_{\perp}^{4}>=8 V_{i}^{4}$ so that Eq. (12) becomes:

$$
\Gamma_{n . l .}(\mu)=\frac{1}{2} \sqrt{\frac{\pi}{2}}\left(\frac{V_{i}}{c}\right)\left(\frac{\Omega_{o}}{\mu}\right) \mathcal{E}(\mu)
$$

where we have made use of the resonant condition Eq. (1). At marginal stability, the growth rate, Eq. (11) and the damping rate, given by Eq. (13), balance each other. Thus, 
by setting $\Gamma_{g}(\mu)=\Gamma_{n . l .}(\mu)$ we obtain an expression for the wave spectrum:

$$
\mathcal{E}(\mu)=A \mu \sqrt{1-\mu^{2}},
$$

where the constant $A$ depends on astrophysical parameters and is defined by:

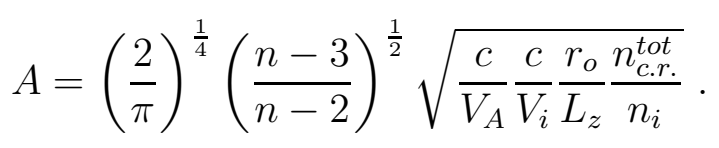

We determine the distribution function from Eq. (6) and Eq. (14):

$$
\frac{\partial \mathcal{F}(\mu)}{\partial \mu}=\frac{4}{\pi}\left(\frac{r_{o}}{L_{z}}\right)\left(\frac{1}{A}\right) \frac{1}{\mu \sqrt{1-\mu^{2}}},
$$

so that as $\mu \rightarrow \mu_{c}, \mathcal{F}(\mu) \propto \ln (\mu)$. For purpose of estimating $\mathcal{E}$ in the coronal regions take $T_{i} \approx 80 \mathrm{eV}, n_{i} \approx 10^{-3} \mathrm{~cm}^{-3}$ and $B_{o} \approx 3 \times 10^{-6} \mathrm{G}$. We than have $\Omega_{o} \approx 5.7410^{-3} \mathrm{rad} / \mathrm{sec}$, $r_{o} \approx 5.23 \times 10^{12} \mathrm{~cm}, V_{i} \approx 8.76 \times 10^{6} \mathrm{~cm} / \mathrm{sec}$ and $V_{A} \approx 2.06 \times 10^{7} \mathrm{~cm} / \mathrm{sec}$. The value of $L_{z}$ is uncertain, and we choose a reasonable value for it, $L_{z} \sim 3 \mathrm{kpc}$. We then have for the astrophysical constant $A \simeq 1.19 \times 10^{-5}$

It is true that if the beat wave amplitude in non-linear damping were too large, thermal particles could become trapped in the wave and the damping rate is reduced. However, in appendix A we show that for the parameters of interest, small angle collisions prevent any such trapping so that Eq. (13) is valid.

\subsection{The Wave Spectrum in the Warm Regions of the I.S.M.}

In the warm regions, neutral atoms are present. The damping rate is then due to charge exchange between the ions and the neutral atoms and is given by (Kulsrud and 
Pearce 1969):

$$
\Gamma_{i n}=\frac{1}{2} n_{H}\langle\sigma v\rangle_{i n},
$$

Therefore, as we will show, in the warm regions $\Gamma_{i n} \gg \Gamma_{n . l .}$ and the marginal stability condition is $\Gamma_{g}(\mu)=\Gamma_{i n}$, where the ion-neutral damping is independent of the pitch angle. Using our expression for the growth rate, Eq. (11), we find that the spectrum is given by the formula:

$$
\mathcal{E}(\mu)=C \mu\left(1-\mu^{2}\right)
$$

where $C$ is:

$$
C=\left(\frac{n-3}{n-2}\right)\left(\frac{c}{V_{A}}\right)\left(\frac{n_{c . r_{.}}^{t o t}}{n_{i}}\right)\left(\frac{r_{o}}{L_{z}}\right) \frac{\Omega_{o}}{2 \Gamma_{i n}} .
$$

By comparing this expression for the wave spectrum with the one obtained using the non-linear Landau damping as the main form of wave dissipation, Eq. (14), we see that the two are very similar, in the sense that both are proportional to $\mu$ as $\mu^{2} \ll 1$ and both are zero at $\mu=1$. The difference appears in the constants $A$ and $C$. Let us then compare then magnitude using typical parameters of the warm regions, $T_{i} \approx 1 \mathrm{eV}, n_{i} \approx 0.03 \mathrm{~cm}^{-3}$ and $B_{o} \approx 3 \times 10^{-6}$ G we have $V_{i} \approx 1 \times 10^{6} \mathrm{~cm} / \mathrm{sec}$ and $V_{A} \approx 3.78 \times 10^{6} \mathrm{~cm} / \mathrm{sec}$. The numerical value for the ion-neutral damping rate is $\Gamma_{i n} \simeq 8.40 \times 10^{-9} n_{H}$ at a plasma temperature of $T_{i} \simeq 10^{4} \mathrm{~K}$ (Kulsrud and Cesarsky 1971). For $n_{H} \approx 0.2 \mathrm{~cm}^{-3}$ and $L_{z}=3 \mathrm{kpc}$, we find that the strength of the magnetic perturbations in the warm regions of the I.S.M. is $C \approx 1.61 \times 10^{-8}$. Note that $C \ll A$, as is expected since the linear damping is more efficient in dissipating the wave energy than the non-linear Landau damping. 
Finally, we remark on the exponential suppression of waves with small wavelength due to their interaction with the thermal ions present in the plasma. That the gap in the wave spectrum generated by ion-cyclotron damping around $\mu=0$ in the absence of mirroring is of the order of $\left(V_{i} / c\right)$ (Holman et al. 1979; Felice 2000). In Sec. 3 we show that this gap does not prevent cosmic rays from crossing the $90^{\circ}$ point since mirror scattering becomes dominant in this region of $\mu$-space. Therefore, we conclude that the overall effect of ion cyclotron damping is not important, although for some time in the past it was a concern.

\section{Scattering Through the $90^{\circ}$ Point - The Adiabatic Region}

From Eq. (16) we see that $F(\mu)$ diverges as $\mu \rightarrow 0$ even if the diffusion coefficient is finite. This result was derived under the assumption that the only wave-particle interaction is the doppler shifted cyclotron resonance $\omega-k_{z} v_{z}=\Omega_{o}$. However, these linearly excited waves have a second order variation in their field strength $\delta B^{2} / B_{o}$ producing a mirror interaction and as $\mu \rightarrow 0$ the pitch angle resonant interaction becomes weak and this mirror interaction takes over. Thus, the theory as given in the previous section is incomplete and this mirror interaction should be added to the pitch angle interaction. In this more complete theory $\partial F(\mu) / \partial \mu$ and $F(\mu)$ are not longer singular at $\mu=0$.

In summary, the growth rate due to the anisotropy decreases as $\mu \rightarrow 0$, while the non-linear damping increases, so that the wave amplitude $\left(\delta B / B_{o}\right)^{2}$ goes as $\mu$. In order to maintain the flux in $\mu$-space $\partial F(\mu) / \partial \mu$ must diverge at $\mu=0$. But if we allow the particles to scatter adiabatically with the long wavelength waves, then we find that the flux in maintained by the mirror interaction so that $F$ is finite as $\mu \rightarrow 0$.

The variation of $\partial F(\mu) / \partial \mu$, which results from the analysis, with and without the mirror interaction in indicated in Fig. (5). The pitch angle below which the mirror 
interaction dominates is denoted by $\mu_{c}$. For much smaller $\mu \approx V_{i} / c \ll \mu_{c}$, the thermal ions can interact with the waves reducing their amplitude even more (Holman et al. 1979), but $F$ is not affected at this smaller $\mu$ since the mirror interaction is mainly carried out by the long wavelength waves which are not affected by the linear cyclotron damping.

The magnetic field can be written as the superposition of the background field plus the Alfven waves, $B=B_{o}+\delta B^{2} / B_{o}$. The adiabatic equation of motion for a particle in the field of linearly polarized Alfven waves (or wave packets of circular polarization) is ( for $\left.k_{\perp}=0\right)($ Krall and Trivelpiece 1990):

$$
\frac{d v_{\|}(t)}{d t}=-\frac{v_{\perp}^{2}}{2 B_{o}} \frac{d}{d z}\left(\frac{\delta B^{2}}{2 B_{o}}\right)
$$

where $\delta B^{2} / 2 B_{o}$ is the perturbed field strength. Let us fix our attention on particles of given $\mu$. Consider all the waves interacting with particles of other pitch angle $\mu_{m}$ (particles with $\mu_{m}$ interact with waves of wavenumber $k_{m}=1 / \mu_{m} r_{o}$ ). In Sec. 2 we showed that $\mathcal{E}(\mu) \propto \mu \propto 1 / k$, so that combined with (20), we see that the mirror force $k_{m} \mathcal{E}\left(\mu_{m}\right)$ is independent of $\mu_{m}$. However, unless the wave packet centered around $k_{m} \propto 1 / \mu_{m}$, is strong enough to mirror the cosmic ray $\mu$, the force on the particle will average out. Thus, it is reasonable to restrict attention to only those waves $\mu_{m}$ whose potential is high enough to reverse the motion of the particle.

Let's rewrite Eq. (20) in terms of a potential as:

$$
\frac{d v_{\|}}{d t}=-\frac{d U(z)}{d z}=-\frac{c^{2}}{4} \frac{d}{d z}\left(\frac{\delta B}{B_{o}}\right)^{2}(z)
$$

where we set $v_{\perp}=c$ (when working near the mirror region we drop $\mu^{2}$ compared to 1 ). A particle which obeys Eq. $(21)$ is reflected (i.e. $\mu \rightarrow-\mu$ ) if the particle parallel energy $v_{\|}^{2} / 2$ is less than the peak of the potential $\mathrm{U}(\max )$. The magnetic field has a wave-like behavior 
and $d / d z \rightarrow k$. The condition for reflection is then:

$$
\mu^{2}<\frac{1}{2}\left(\frac{\delta B}{B_{o}}\right)^{2}=\frac{\mathcal{E}\left(\mu_{m}\right)}{2}
$$

A word on our notation. We introduce the notation $\mu_{m}$ to distinguish the particle $\mu$ which is being mirrored from the wave which is doing the mirroring. The mirroring wave is label by its wavenumber $k_{m}$ and it is created by the particle $\mu_{m}$ through the resonance condition $k_{m}=1 / \mu_{m} r_{o}$. This is equivalent to parameterizing the wavelength by $\mu_{m}$. This is convenient because the adiabatic interaction is not a resonant interaction. In the quasi-linear region, where the scattering is of a resonant type, for every particle $\mu$ there is only one resonating wavelength $\lambda=2 \pi \mu r_{o}$, but in the adiabatic region a particle with a given $\mu \ll 1$ interacts with waves of long wavelength (i.e. those waves which are resonant with $\mu \sim 1$ particles). From Eq. (22) we see that for fixed $\mu_{m}$ there is a maximum $\mu$ below which reflection becomes possible. This particular value of $\mu$ that we shall denote by $\mu_{c}$ corresponds to adiabatic interaction of particles with the largest wavelength waves present in the system, those corresponding to $\mu_{m} \sim 1$. Therefore:

$$
\mu_{c}=\sqrt{\frac{A}{2}} .
$$

If we use the value for $A$ obtained in Sec. $2, A \simeq 1.19 \times 10^{-5}$, we than have $\mu_{c} \simeq 2.44 \times 10^{-3}$. In the warm regions of the interstellar space the critical value $\mu_{c}$ is still given by the above expression with $A$ replaced by $C$. Using our previous result, $C \approx 1.61 \times 10^{-8}$, and $\mu_{c} \simeq 8.97 \times 10^{-5}$. Therefore, we see that in the coronal region, where ion-cyclotron damping at small wavelengths occurs, the dissipation region where the waves are exponentially damped, $\mu \lesssim V_{i} / c$, is smaller than the onset of the adiabatic region. 


\subsection{The Matching between the Adiabatic Region and the Quasi-Linear Region}

We now write down the equations for the cosmic ray distribution function $F$ and for the wave spectrum $\mathcal{E}=\left(\delta B / B_{o}\right)^{2}$ in the boundary layer region. In steady state, the cosmic ray distribution function $F(\mu, z, p)$ satisfies Eq. (2), where now the term corresponding to the mirror interaction, the $\dot{\mu}$ term, becomes important. From the mirror equation, Eq. (20), we obtain the expression for $\dot{\mu}$ :

$$
\dot{\mu}(z)=-\frac{\Omega}{2} \mathcal{E}_{m} \cos \left(\frac{z}{r_{o}}\right) .
$$

where we take the magnetic field to have a sinusoidal behavior: $\left(\delta B / B_{o}\right)^{2}=\mathcal{E} \cos (k z)$ and $\mathcal{E}_{m}$ is the wave spectrum evaluated at $\mu_{m}=1$. We normalize Eq. (2) together with the marginal stability condition in the boundary layer region to obtain:

$$
\begin{aligned}
& \tilde{\mu} \frac{\partial \tilde{\mathcal{F}}}{\partial \tilde{z}}-\cos (\tilde{z}) \frac{\partial \tilde{\mathcal{F}}}{\partial \tilde{\mu}}=\frac{\pi}{4} \frac{\partial}{\partial \tilde{\mu}}\left[\tilde{\mathcal{E}} \frac{\partial \tilde{\mathcal{F}}}{\partial \tilde{\mu}}\right], \\
& \frac{\partial \tilde{\mathcal{F}}}{\partial \tilde{\mu}}=\frac{\tilde{\mathcal{E}}}{\tilde{\mu}^{2}} .
\end{aligned}
$$

where we have defined the normalized quantities $\tilde{\mu}=\mu / \mu_{c}$ and $\tilde{z}=z / r_{o}$, together with $\tilde{\mathcal{E}}=\mathcal{E} / A \mu_{c}$ and $\tilde{\mathcal{F}}=\mathcal{F} / \mathcal{F}_{c}$, with

$$
\mathcal{F}_{c}=\frac{4}{\pi}\left(\frac{r_{o}}{L_{z}}\right)\left(\frac{1}{A}\right) \simeq 1.8 \sqrt{\frac{V_{A}}{c} \frac{V_{i}}{c} \frac{r_{o}}{L_{z}} \frac{n_{i}}{n_{c . r .}^{t o t}}} .
$$

where we used the definition of $A$ as given by Eq. (15) and we evaluated the numerical constant using $n=4.7$. Deep in the quasi-linear region $(\tilde{\mu} \gg 1)$ the cosmic ray distribution

function is $\tilde{\mathcal{F}}(\tilde{\mu})=\ln (\tilde{\mu})+1$. As we move closer to the adiabatic region from the quasi-linear 
region, the term corresponding to the mirror interaction becomes important. In order to solve the system of equations numerically, we seek the correct boundary condition near $\tilde{\mu} \gtrsim 1$, where a perturbation approach in powers of $1 / \tilde{\mu}$ is valid. This is:

$$
\tilde{\mathcal{F}}(\tilde{\mu}, \tilde{z}) \simeq \ln (\tilde{\mu})+1+\frac{\sin (\tilde{z})}{\tilde{\mu}^{2}}-\frac{2 \pi \cos (\tilde{z})+\sin ^{2}(\tilde{z})}{\tilde{\mu}^{4}}+O\left(\frac{1}{\mu^{6}}\right)
$$

In the next subsection we numerically solve the system of partial differential equations, Eqs. (25) and Eqs. (26) subject to the boundary conditions given by Eq. (28). The contribution to the drift velocity, Eq. (8), coming from the $0 \leq \mu \lesssim \mu_{c}$ region only involves a dimensionless constant times the normalization. Therefore, the remainder of this Section is devoted to the numerical solution of the boundary layer problem which will ultimately give us the numerical value of the constant. The numerical solution also elucidate the physics of the mirror scattering through the $90^{\circ}$ point.

\subsection{Numerical Solution of the Boundary Layer}

We solve the dimensionless system of equations, Eqs. (25) and (26), for the cosmic ray distribution function $\tilde{\mathcal{F}}(\tilde{\mu}, \tilde{z})$ and the spectrum $\tilde{\mathcal{E}}(\tilde{\mu}, \tilde{z})$ by a finite difference method. In a way this problem is very similar to a boundary layer problem in which the global solution of a system of equations in known everywhere except in a small region in space. The global solution is then used as a boundary condition for the boundary layer which itself is investigated numerically by integrating the equations subject to the boundary conditions imposed by the global solution.

We create a grid in $(\tilde{\mu}, \tilde{z})$ space and write Eqs. (25) and Eqs. (26) as difference

equations. We fix the boundary condition on $\tilde{\mathcal{F}}$ by Eq. (28) at a value of $\mu$ large enough to justify the expansion. We choose $\tilde{\mu}= \pm 4$. We then implement periodic boundary condition 
in $\tilde{z}$ such that $\tilde{\mathcal{F}}(\tilde{\mu}, \tilde{z}=0)=\tilde{\mathcal{F}}(\tilde{\mu}, \tilde{z}=2 \pi)$. We solve the system of differential equations using the relaxation method. Both the finite difference method and the relaxation method used to solve systems of P.D.E. with fixed boundary conditions are discussed extensively in the literature (see for example Press et. al. (1992)) and they will not be described in this paper.

The quasi-linear solution is odd-symmetric in $\mu$ so that $\tilde{\mathcal{F}}(\tilde{\mu}=4, \tilde{z})=-\tilde{\mathcal{F}}(\tilde{\mu}=-4, \tilde{z})$. We specify an initial form for the distribution function $\tilde{\mathcal{F}}(\tilde{\mu}, \tilde{z})$ and the spectrum $\tilde{\mathcal{E}}(\tilde{\mu}, \tilde{z})$ and let it relax in time until the solution converges and the final answer becomes time independent. Note that both the distribution function and the spectrum now depends on $z$, i.e. the mirroring depends on where the particle is found within the wave packet. We assume that locally (in $z$ ) the growth and the damping balance each other so that a steady state can be reached and a well defined wave spectrum created (indeed, due to Alfven wave dispersion, local growth and damping of the wave happens in a time scale shorter compared to the time wave packets propagate relative to the long wave (Foote and Kulsrud 1979)) .

Before we present the answer let us describe qualitatively what happens as a particle approaches the adiabatic region from the quasi-linear region. The particle energy is the sum of the kinetic energy and the potential energy of the magnetic field:

$$
\frac{\tilde{\mu}^{2}}{2}+\sin (\tilde{z})=\text { const. }=E
$$

where $E$ is the energy of the particle, which is obviously conserved in the absence of quasilinear scattering. In Fig 1 we presents the contour plots of Eq. (29) for different values of the energy $E$. Note the presence of a separatrix at $E=1$ and the trap region for $E<1$. In the quasi-linear region (large $\mu$ ) the lines are only weakly distorted from straight lines. In this region the distribution function is independent of $z$ and the scattering is mostly resonant (the mirroring being small). As a particle moves closer to the separatrix, 
its motion will be determined by the combined effect of the mirroring and the resonant diffusion. The distribution function $\tilde{\mathcal{F}}$ will then depend on $z$, i.e. the motion of the particle will depend on where in the potential well of the wave the particle is located. Note that from our scaling condition we found that the various terms of the cosmic ray diffusion equation, which are the convection term, the mirror term and the diffusion term, are all comparable in strength at $\mu=\mu_{c}$. Thus, deep in the mirror region there is still a finite energy in the waves which can resonant scatter the particles and force them to change their orbit as they move in the potential well of the magnetic field in the adiabatic motion.

To carry out the numerical integration we create a grid $\left(I_{\max } \times J_{\max }\right)$ in $(\tilde{\mu}, \tilde{z})$ space for $-4 \leq \tilde{\mu} \leq 4$ and $0 \leq \tilde{z} \leq 2 \pi$. We find that a grid with $I_{\max }=100$ and $J_{\max }=50$ is fine enough to represents the differential equations by the difference equations (that is to say, as we increase the grid resolution the solution does not change significantly). We choose our time step to be small enough to ensure stability and convergence. In these particular runs we have chosen the increments for the various variables to be $d \tilde{\mu}=8 / 100=0.080, d \tilde{z}=2 \pi / 50=0.125$ and $d \tilde{t}=d \tilde{\mu}^{2} / 16=0.0004$. The typical relaxation time is $\tau_{\text {relax }}=1 / d \tilde{t}=2500$. We find that the solution approaches steady state after few relaxation times. We can see this in Fig 2 where we show the distribution function as a function of time for 4 different points $(\tilde{\mu}, \tilde{z})$ as a function of the relaxation time. Notice that the functions become constant after few relaxation times. Therefore, out final solution is time independent and represents the correct solution of the system of equations (25) and $(26)$.

In Fig. 3 we show the plot of the normalized cosmic ray distribution function $\tilde{\mathcal{F}}(\tilde{\mu}, \tilde{z})$ as a function of $\mu / \mu_{c}$ for different values of $\tilde{z}$. Note that the curves all approach the quasi-linear solution as $\tilde{\mu} \rightarrow \pm 4$, that is, $\tilde{\mathcal{F}} \sim \ln (\tilde{\mu})$. Note also how the solution if well behaved as $\tilde{\mu} \rightarrow 0$. Inside the boundary layer the distribution function is not necessarily 


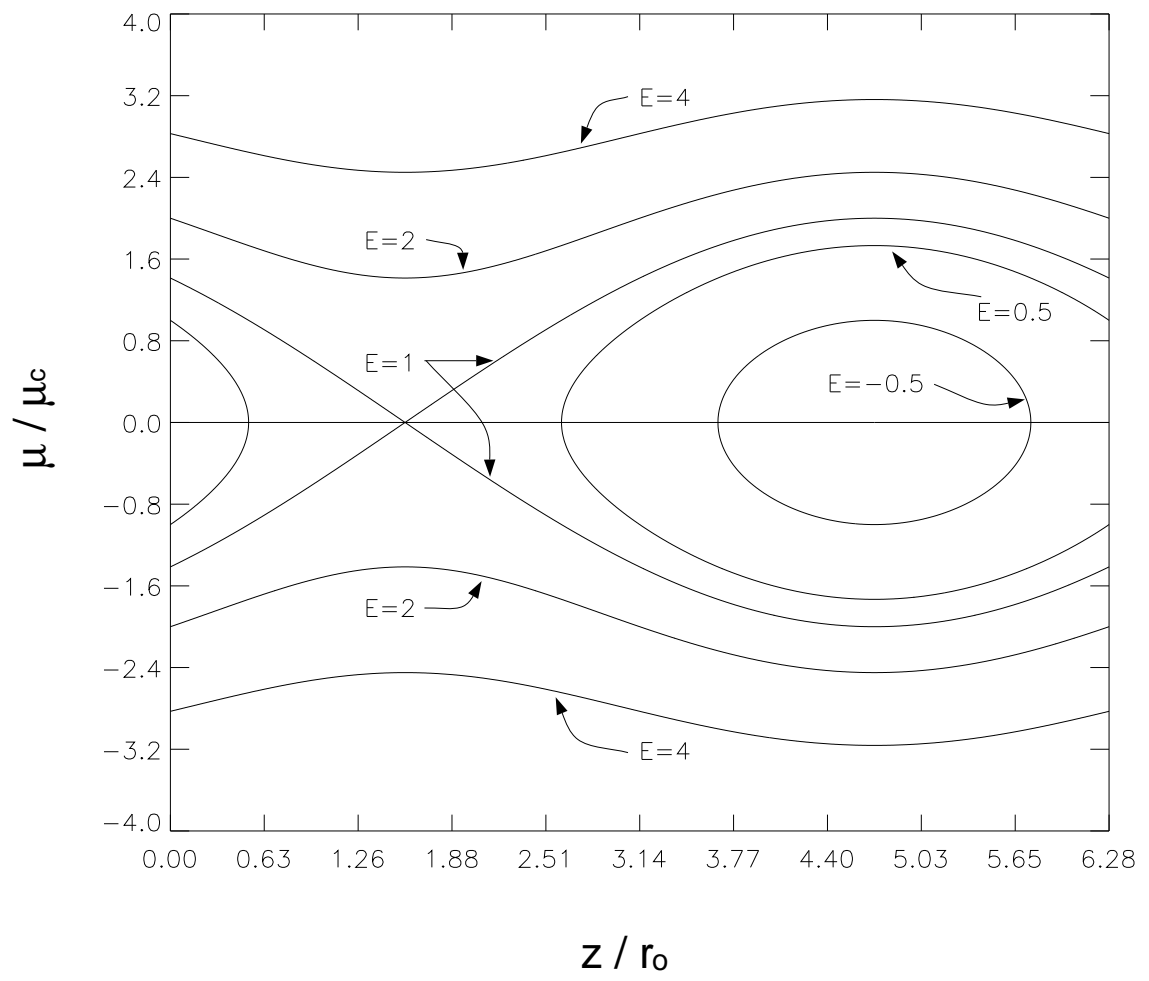

Fig. 1. - Contour Plots of the Particle Energy. Note the presence of a separatrix at $E=1$ and the trap region for $E<1$ 


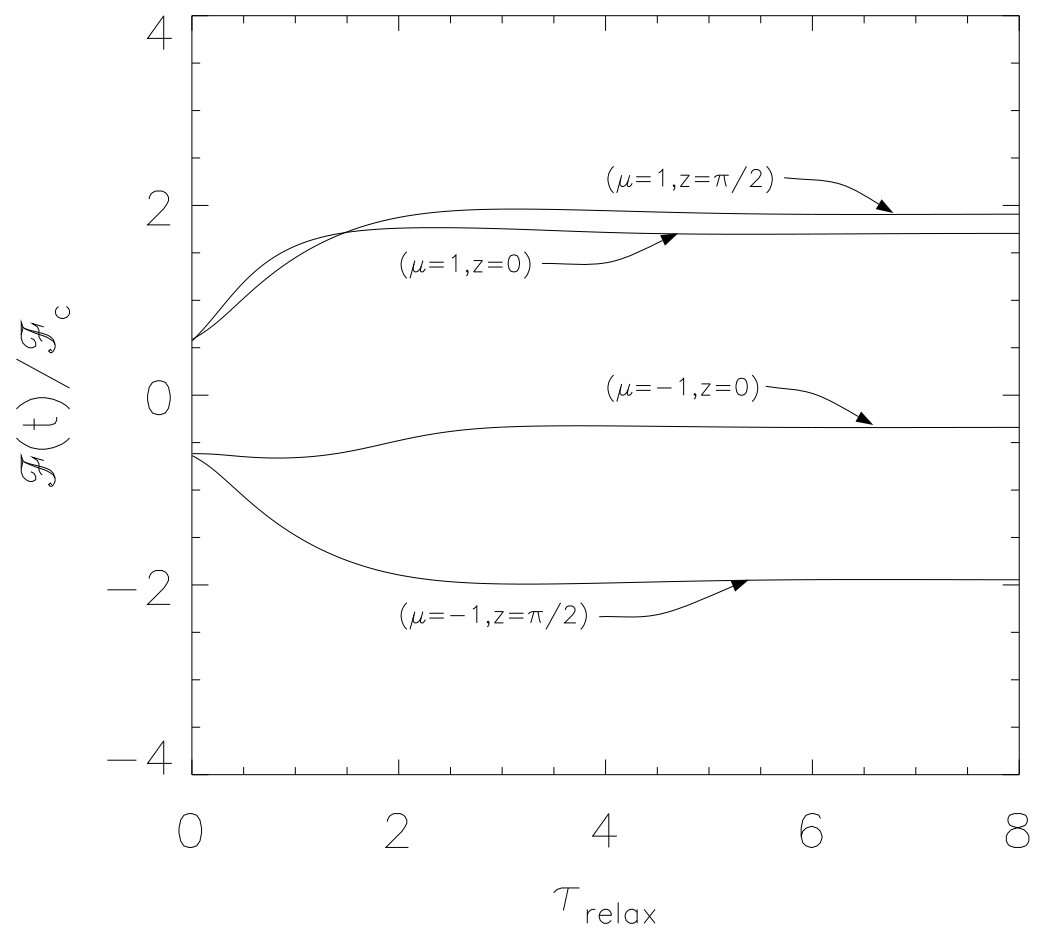

Fig. 2.- In this plot we show the convergence of the solution to the steady state cosmic ray distribution function in the adiabatic region for four different points in $(\tilde{\mu}, \tilde{z})$. Notice how the distribution function becomes a constant after few relaxation times 
odd-symmetric for all values of $\tilde{z}$ even though the equations themselves are symmetric. In Fig 4 we present the plots of the wave spectrum $\tilde{\mathcal{E}}(\tilde{\mu}, \tilde{z})$ as a function of $\tilde{\mu}$ for two values of $\tilde{z}$. Note that the wave spectrum is perfectly symmetrical.

Finally, in Figs. 5 and 6 we present the averaged values for the distribution function and of the wave spectrum defined as:

$$
\langle\tilde{\mathcal{F}}(\tilde{\mu}, \tilde{z})\rangle_{\tilde{z}}=\frac{1}{2 \pi} \int_{0}^{2 \pi} d \tilde{z} \tilde{\mathcal{F}}(\tilde{\mu}, \tilde{z})
$$

and likewise for $\langle\tilde{\mathcal{E}}(\tilde{\mu}, \tilde{z})\rangle$. Note that this averaged solution for the distribution function is both odd-symmetric and well behaved around $\mu=0$. In this plot we also present the quasi-linear solution given by $\ln (\tilde{\mu})+1$. It is clear that the analytical solution approaches the numerical solution as one moves away from the adiabatic region. It is striking to see what difference the mirror interaction has on the distribution function. Indeed, one can see how without the mirror term one would get a singularity at $\mu=0$ which is removed by the inclusion of the adiabatic effect.

\subsubsection{The Cosmic Rays Drift Velocity}

We can now evaluate the cosmic ray parallel flux and their drift velocity given by Eq. (8). The integral must be divided into two parts, one corresponding to the contribution coming from the adiabatic region and the other coming from the quasi-linear region. Thus, in terms of $\tilde{\mu}$

$$
\frac{V_{d r i f t}}{c}=2 \mu_{c}^{2} \mathcal{F}_{c}\left[\int_{0}^{4} d \tilde{\mu} \tilde{\mu}\langle\tilde{\mathcal{F}}(\tilde{\mu}, \tilde{z})\rangle_{z}+\int_{4}^{1 / \mu_{c}} d \tilde{\mu} \tilde{\mu} \tilde{\mathcal{F}}(\tilde{\mu})\right]
$$

where 4 is the maximum value of $\tilde{\mu}$ used in the numerical solution of $\tilde{\mathcal{F}}(\tilde{\mu}, \tilde{z})$ and $\langle\tilde{\mathcal{F}}(\tilde{\mu}, \tilde{z})\rangle_{\tilde{z}}$ 


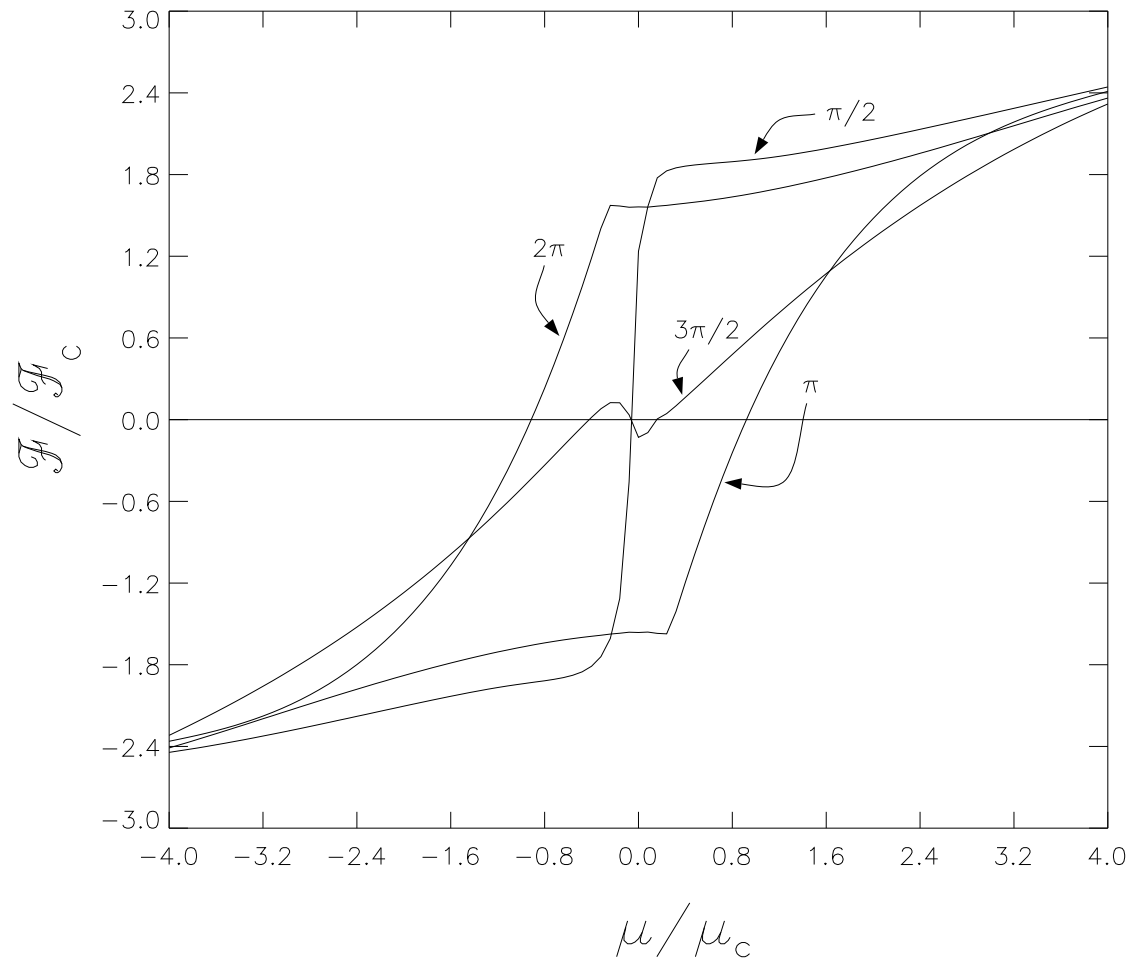

Fig. 3.- Plots of the distribution function $\tilde{\mathcal{F}}(\tilde{\mu}, \tilde{z})$ in the adiabatic region for $\tilde{z}=$ $\pi / 2, \pi, 3 \pi / 2,2 \pi$ 


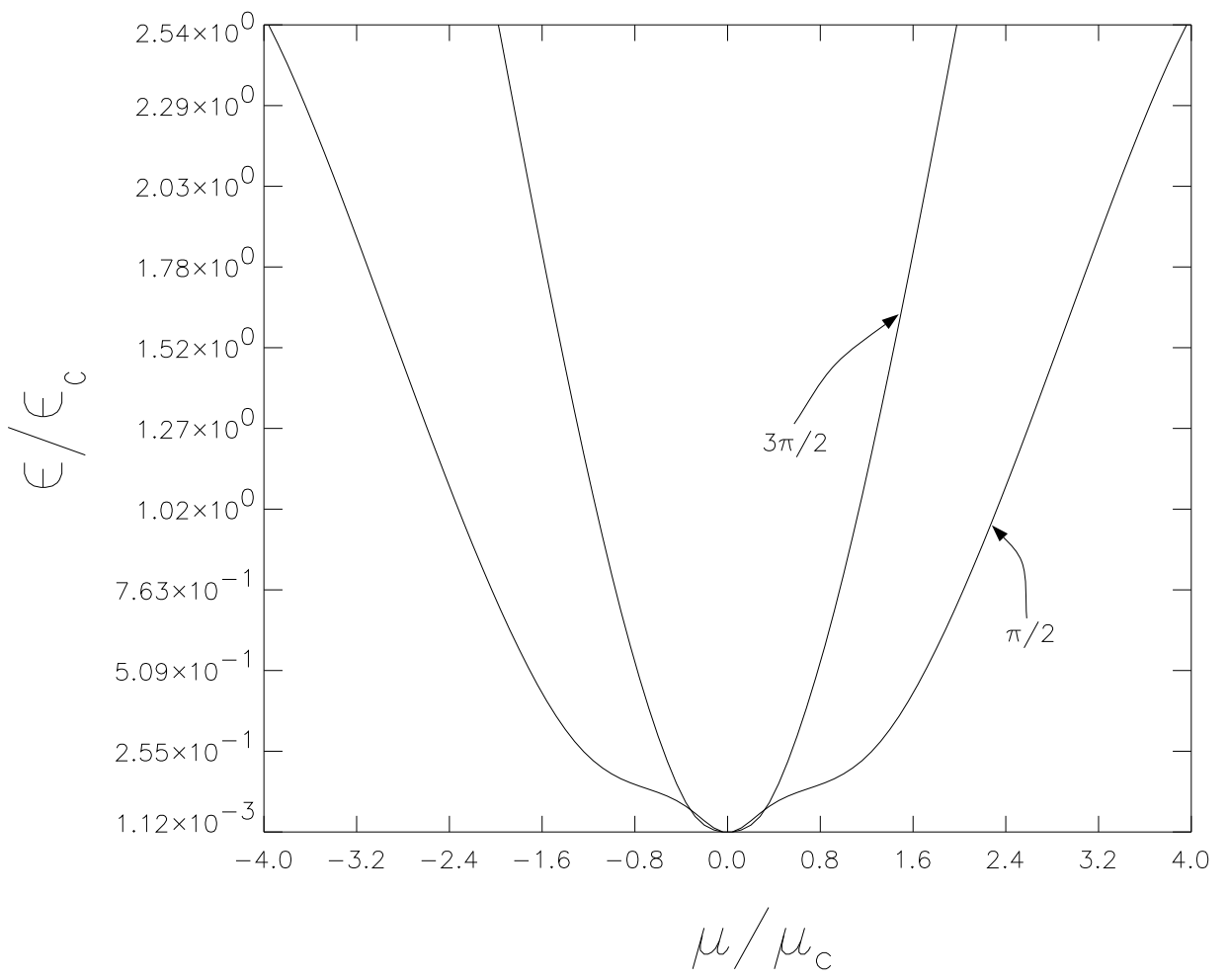

Fig. 4.- Plots of the normalized wave spectrum $\tilde{\mathcal{E}}(\tilde{\mu}, \tilde{z})$ in the adiabatic region for $\tilde{z}=$ $\pi / 2,3 \pi / 2$ 


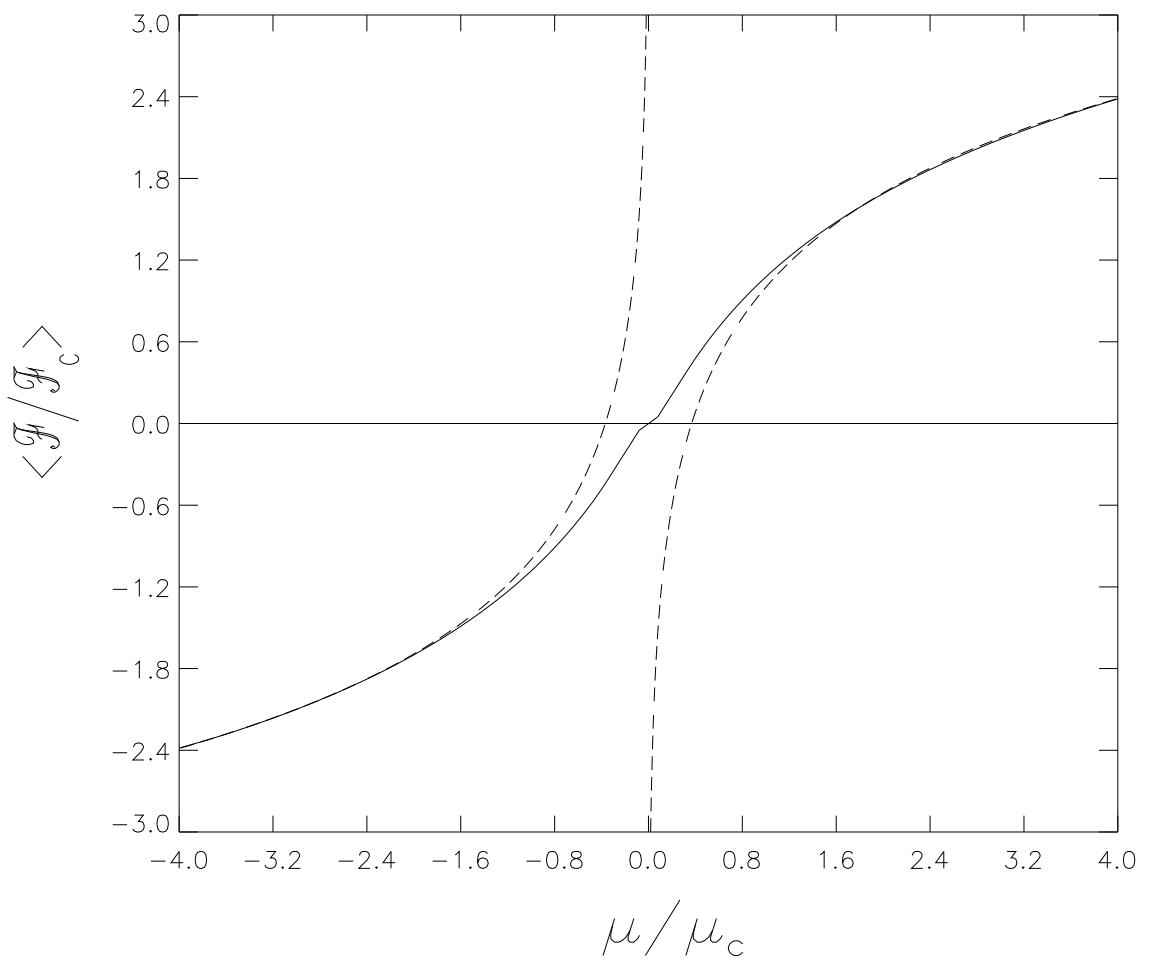

Fig. 5. - Plots of the averaged normalized distribution function $\langle\tilde{\mathcal{F}}(\tilde{\mu}, \tilde{z})\rangle_{z}$ in the adiabatic region 


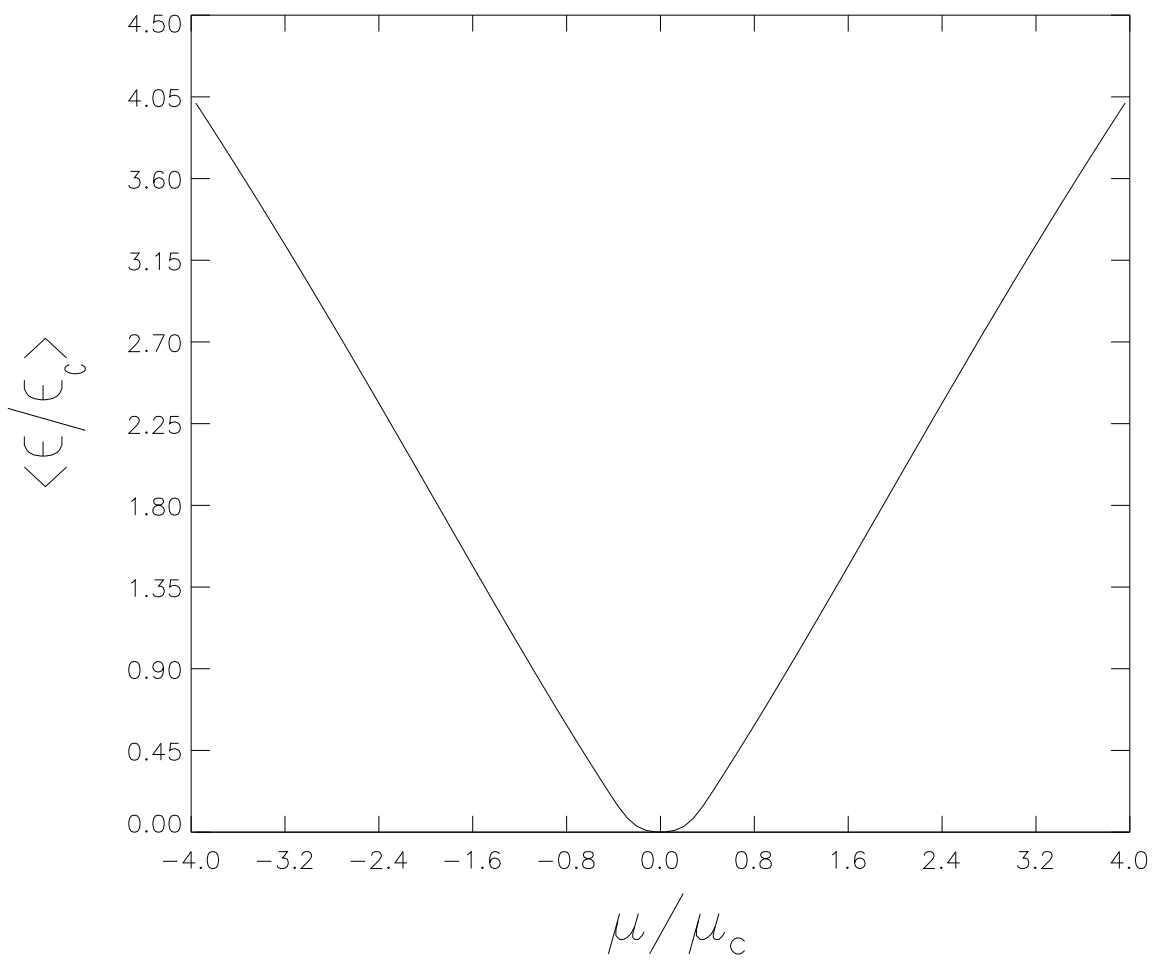

Fig. 6. - Plots of the averaged normalized wave spectrum $\langle\tilde{\mathcal{E}}(\tilde{\mu}, \tilde{z})\rangle_{z}$ in the adiabatic region 
is given by Eq. (30) and plotted in Fig. 5. Upon numerical integration we find:

$$
\int_{0}^{4} d \tilde{\mu} \tilde{\mu}\langle\tilde{\mathcal{F}}(\tilde{\mu}, \tilde{z})\rangle_{z} \approx 1.90
$$

Eq. (32) represents the normalized flux carried by the mirroring in the adiabatic region. Using the quasi-linear solution for the distribution function, $\tilde{\mathcal{F}}=\ln (\tilde{\mu})+1$, we can evaluate the second integral in Eq. (31) to obtain the final expression for the cosmic ray diffusion coefficient. We can also express our result in terms of the cosmic ray anisotropy $\delta$ and diffusion coefficient $\mathcal{D}$ by means of the relation $\delta=3 \mathcal{D} / c L_{z}$, where the cosmic ray drift velocity is $V_{\text {drift }}=\delta c / 3$. Then in the rest frame we obtain:

$$
\frac{V_{\text {drift }}-V_{A}}{c}=\mathcal{F}_{c}\left\{\left[\ln \left(\frac{1}{\mu_{c}}\right)+\frac{1}{2}\right]-26.37 \mu_{c}^{2}\right\}
$$

where the expression for $\mathcal{F}_{c}$ is given by Eq. (27). Note that the contribution to the cosmic ray diffusion coefficient coming from the adiabatic region is small compared to the contribution coming from the quasilinear region (essentially by $\mu_{c}^{2}$ ). This is because the boundary layer region $\left(0 \leq \mu \lesssim \mu_{c}\right)$ is much smaller than the quasilinear region $\left(\mu_{c} \lesssim \mu \leq 1\right)$. On the other hand, this region is very important since it sets the lower limit cutoff to the quasi-linear region $\mu_{c}$ and thus effects the magnitude of the cosmic ray diffusion coefficient.

We can estimate the numerical value of the diffusion coefficient using the typical values for the various physical parameter presented above. In the coronal region, where the main form of the damping mechanism is non-linear Landau damping, we find $\mathcal{F}_{c} \simeq 6.03 \times 10^{-5}$ and $\mu_{c} \simeq 2.44 \times 10^{-3}$. The Alfven velocity is $V_{A} \approx 2.06 \times 10^{7} \mathrm{~cm} / \mathrm{sec}$ and the cosmic ray drift velocity in the rest frame is then $V_{\text {drift }} \approx 3.24 \times 10^{7} \mathrm{~cm} / \mathrm{sec}$. This corresponds to a value for the cosmic ray diffusion coefficient of $\mathcal{D} \simeq 2.99 \times 10^{29} \mathrm{~cm}^{2} / \mathrm{sec}$ and a value for the 
cosmic ray anisotropy of $\delta \simeq 3.24 \times 10^{-3}$. Thus, from our analysis it is evident that in the coronal region the cosmic rays are very efficiently scattered by the hydro-magnetic waves they generate so that they are essentially convected at the local Alfven speed.

In the warm HI regions, where the main damping mechanism is due to ion-neutral charge exchange, the diffusion coefficient can be substantially higher, since the dissipation mechanism is more severe. The analysis which leads to the expression for $\mathcal{D}$ in the warm region is the same as the one followed to find the diffusion coefficient in the coronal region, accounting for the difference in the energy in the waves. With the values of parameters presented above, we find $\mathcal{F}_{c} \simeq 4.47 \times 10^{-2}$ and $\mu_{c} \simeq 8.97 \times 10^{-5}$. The Alfven velocity is $V_{A} \approx 3.78 \times 10^{6} \mathrm{~cm} / \mathrm{sec}$ and the cosmic ray drift velocity in the rest frame is then $V_{\text {drift }} \approx 10^{10} \mathrm{~cm} / \mathrm{sec}$. Note that the factor introduced by the logarithm of $1 / \mu_{c}$ is of the order of 10 in the warm region.

According to our analysis, cosmic rays can propagate through the warm regions with drift velocity comparable to the speed of light. This is the result of a very strong linear damping and the impossibility for the waves to growth at large enough amplitude where they could scatter the particles. However, this large anisotropy and drift velocity in the warm region is actually found to be much smaller when it is appreciated that the warm regions are mixed in with the coronal regions, where the drift velocity is few times the Alfven speed. In fact the cosmic ray density is essentially constant in the warm regions (its effective density gradient scale length $L_{z}$ is very large). The effective length in the coronal region is the true length of the lines in the coronal regions only (see Figure 7). The observed anisotropy in the warm regions is then given by the anisotropy in the coronal regions.

Finally, we can now calculate the cosmic ray residence time in the gaseous disk. The particle confinement in the disk is mainly accomplished in the hot coronal regions, as demonstrated above. Thus, the cosmic ray residence time can be estimated by: 


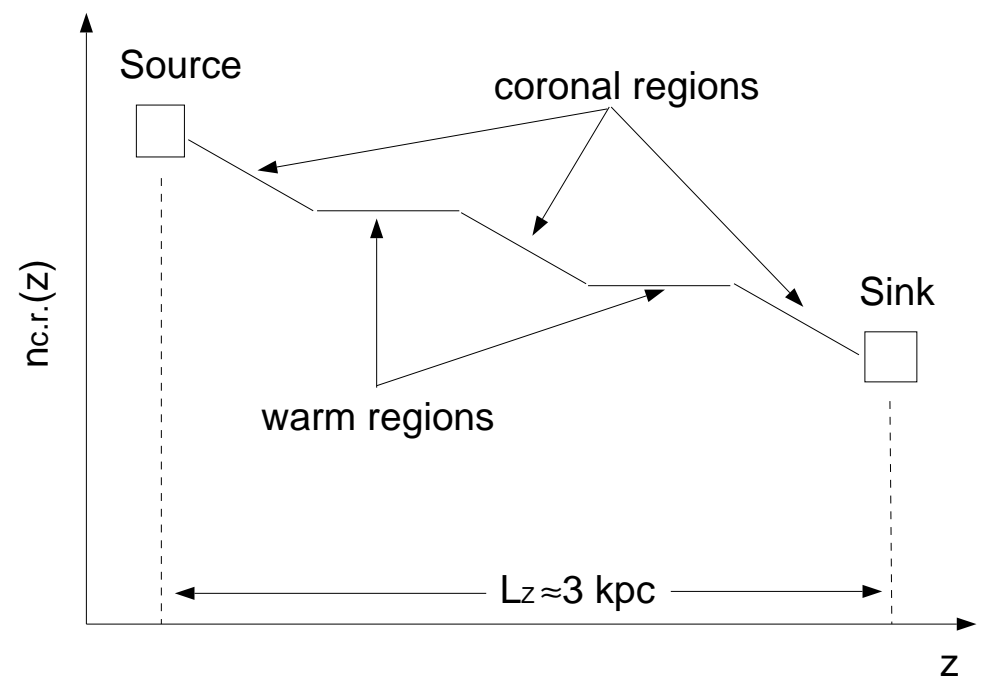

Fig. 7.- Effective Cosmic Rays Density Gradients in the I.S.M. The density gradient in the warm regions is effectively infinite (and the particle density constant) 


$$
t_{\text {life }}=f_{h}\left(\frac{L_{z}}{V_{\text {drift }}}\right)
$$

where $f_{h}$ is the fraction of the disk that is filled with hot plasma and $V_{\text {drift }} \approx 3.24 \times 10^{7} \mathrm{~cm} / \mathrm{sec}$ is the cosmic ray drift velocity in the coronal regions as estimated above. Taking $f_{h}=1 / 2$ and $L_{z}=3 \mathrm{kpc}$ one then finds that $t_{\text {life }} \approx 4.5$ Myrs, which is of the same order of the three million years derived from the chemical composition. In Sec. 5 we discuss this result and its implications. However, first we derive the energy dependence of the cosmic ray diffusion coefficient which is done in the next section.

\section{Energy Dependence of the Diffusion Coefficient}

In Sections 2 and 3 we focused our attention on the pitch angle scattering of cosmic rays of a few $\mathrm{GeV}$. We now wish to consider the general problem of the energy dependence of the diffusion coefficient. In calculating the growth rate we must include the contribution coming from particles of all energies to the growth of waves with given wavenumber $k$. The growth rate then becomes:

$$
\Gamma_{g}(k)=2 \pi^{2} e^{2} \frac{V_{A}}{c} \frac{1}{k} \int_{p_{o}}^{\infty} d p p \int_{-1}^{1} d \mu\left(1-\mu^{2}\right) \frac{\partial F(p, \mu)}{\partial \mu} \delta\left(\mu-\frac{p_{*}}{p}\right)
$$

where $p_{*}=e B_{o} / c k$.

There are two ranges of $k$ to consider; First, if $p_{*}<p_{o}$ (short wave lengths, the $\mu \gamma<1$ region in Fig. 8) then there is a resonant $\mu$ for all $p>p_{o}$. Second, if $p_{*}>p_{o}$ (long wave lengths, the $\mu \gamma>1$ region in Fig. 8), then there is a resonant $\mu$ only for $p>p_{*}$.

Thus, in the first case, the $p$ integral extends from $p_{0}$ to $\infty$ and we obtain 


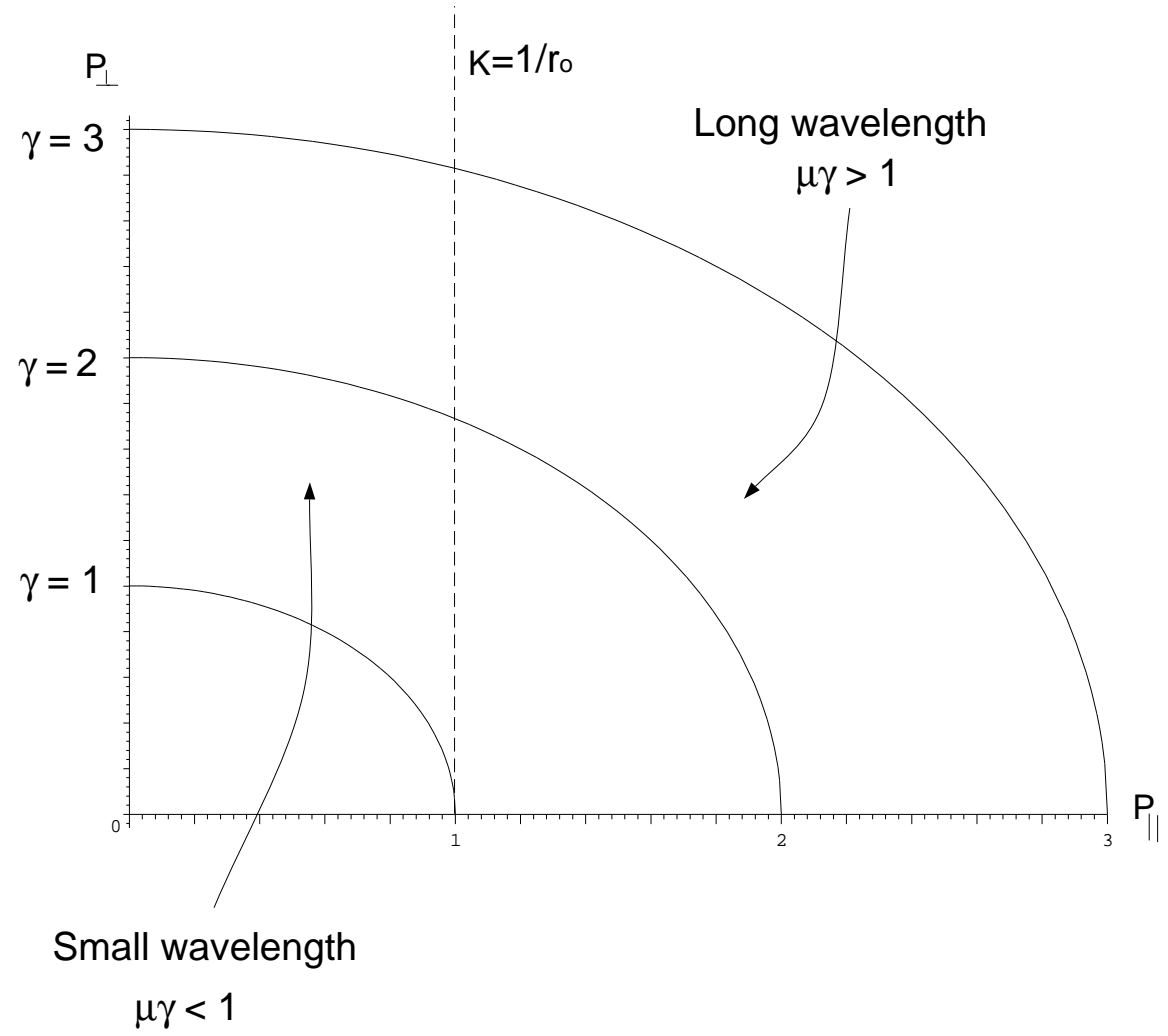

Fig. 8.- Resonant surfaces of constant $k=e B / c p_{*}$. Here $p_{*}=\mu p=p_{z}$ is the parallel projection of the cosmic ray momentum along the magnetic field 


$$
\Gamma_{g}=\frac{\Omega_{o}}{2}\left(\frac{c}{V_{A}}\right)\left(\frac{n_{c . r .}^{t o t}}{n_{i}}\right)\left[1-\frac{n-3}{n-1}\left(\frac{p_{*}}{p_{o}}\right)^{2}\right] \frac{1}{L_{z} k} \frac{1}{\mathcal{E}(k)} \quad \text { for } k>e B_{0} / c p_{0}
$$

In the second case, $p_{*}>p_{0}$ the $p$ integral extends from $p_{*}$ to $\infty$ and we obtain

$$
\Gamma_{g}=\frac{\Omega_{o}}{2}\left(\frac{c}{V_{A}}\right)\left(\frac{n_{c . r .}^{*}}{n_{i}}\right)\left[1-\frac{n-3}{n-1}\right] \frac{1}{L_{z} k} \frac{1}{\mathcal{E}(k)} \quad \text { for } k<e B_{0} / c p_{0}
$$

where $n_{c . r .}^{*}=n_{c . r .}^{\text {tot }}\left(p_{o} / p_{*}\right)^{n-3}$ is the number of cosmic rays with energy above $p_{*}$. Note that if $p_{*}=p_{o}$, Eqs. (36) and (37) are the same.

The damping rates, both in the coronal regions and in the warm HI regions, are the same as given by Eq. (12) and Eq. (17). As before, we assume marginal stability of the waves so that by equating the growth rate to the damping rates we obtain the wave spectrum. We neglect constant terms of order one.

In the coronal regions of the disk, where the main form of damping is non-linear Landau damping, we obtain:

$$
\mathcal{E}(\mu, \gamma) \simeq \begin{cases}A \mu^{0.15} \gamma^{0.15} & \text { if } p_{*}<p_{o} \\ A \mu \gamma & \text { if } p_{*}>p_{o}\end{cases}
$$

where $A$ is still defined by Eq. (15). (Since for resonance $\gamma \mu=e B_{0} / m c^{2} k, \mathcal{E}(\mu \gamma)$ is really a function of the single variable $k$.)

In the HI regions, where the main form of damping is ion-neutral charge exchange, we obtain:

$$
\mathcal{E}(\mu, \gamma) \simeq \begin{cases}C \mu^{-0.7} \gamma^{-0.7} & \text { if } p_{*}<p_{o} \\ C \mu \gamma & \text { if } p_{*}>p_{o}\end{cases}
$$


where $C$ is given by Eq. (19). Note that the spectrum is continuous at the transition point $\mu \gamma=1$.

In our $\gamma=1$ analysis in Sec. 3 we found that the energy dependence of the overall diffusion coefficient is dominated by the quasi-linear region and its cutoff $\mu_{c}$. Since the correction due to the mirror region appears as the log of $\mu_{c}$, we can safely neglect its energy dependence. Then from Eq. (8),

$$
\mathcal{D}(\gamma) \approx v^{2} \int_{\mu_{c}}^{1} \frac{\left(1-\mu^{2}\right)}{\nu(\mu, \gamma)} d \mu
$$

If $\gamma>1$, the integral must be broken into two parts. From Eq. (39, for $\mu<1 / \gamma, \mathcal{E}=A \mu \gamma$, and for $\mu>1 / \gamma, \mathcal{E}=A \mu^{0.15} \gamma^{0.15}$. We then find:

$$
\mathcal{D}(\gamma) \simeq \frac{4}{\pi} \frac{c^{2}}{\Omega_{o}} \frac{1}{A}\left\{\left[\ln \left(\frac{1}{\mu_{c} \gamma}\right)-\frac{1}{2 \gamma}\left(1-\mu_{c}^{2} \gamma^{2}\right)\right]+\left[0.83 \gamma^{0.85}-1.18+\frac{0.35}{\gamma^{2}}\right]\right\}
$$

where the first term in parenthesis must be set to zero when $\gamma \geq 1 / \mu_{c} . \mu_{c}$ is given by Eq. (23) in the coronal region. In Fig. 9 we plot this expression for $\mu_{c}=2.13 \times 10^{-3}$. Note that for $\gamma_{c} \gtrsim 7$ the diffusion coefficient has the power law energy dependence $\mathcal{D} \propto \gamma^{0.85}$. Also note how the energy dependence deviates strongly from the simple power law for small values of the cosmic ray energy.

In the warm regions the energy dependence of the diffusion coefficient is readily calculated using Eq. (8) together with the form of the spectrum given by Eq. (38). Thus, the expression for $\mathcal{D}(\gamma)$ in the warm regions of the interstellar space is:

$$
\mathcal{D}(\gamma) \simeq \frac{4}{\pi} \frac{c^{2}}{\Omega_{o}} \frac{1}{C}\left\{\left[\ln \left(\frac{1}{\mu_{c} \gamma}\right)-\frac{1}{2 \gamma}\left(1-\mu_{c}^{2} \gamma^{2}\right)\right]+\left[0.32 \gamma^{1.7}-0.59+\frac{0.27}{\gamma^{2}}\right]\right\}
$$




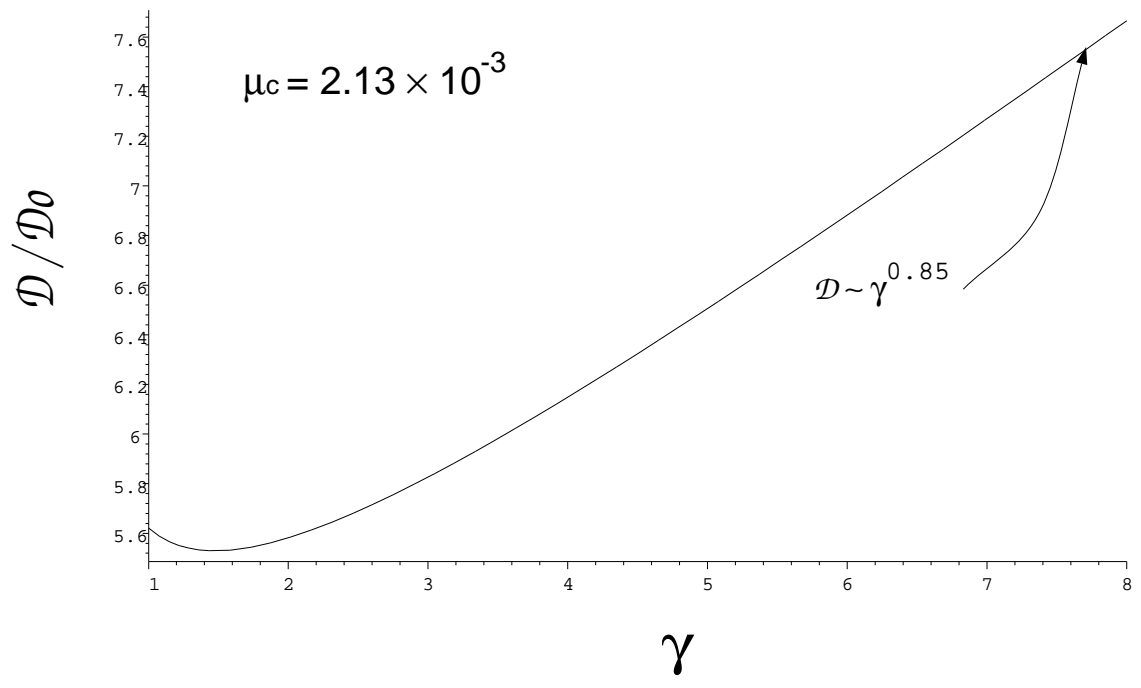

Fig. 9. - Plot of the normalized diffusion coefficient as a function of energy for $\mu_{c}=2.2 \times 10^{-3}$ 
where $\mu_{c}=\sqrt{C / 2}$. In this region the diffusion coefficient very similar to the diffusion coefficient in the coronal regions, Eq. (41), with the difference that now the energy dependence is much stronger. Indeed, we have that in the warm regions of the interstellar space $\mathcal{D} \propto \gamma^{1.7}$.

Finally, it must be noted that the energy dependence for the diffusion coefficient in

the coronal region, $\mathcal{D} \propto \gamma^{0.85}$, at large energies, is the same as that found in Cesarsky and Kulsrud (1981) and the energy dependence for the diffusion coefficient in the warm regions, $\mathcal{D} \propto \gamma^{1.7}$ is the same as they found in Kulsrud and Cesarsky (1971). In this Section we have extended these earlier results to cover the correction due to the presence of the mirror region which becomes important at energies of a few $\mathrm{GeV}$.

\section{Conclusions}

In this paper we studied cosmic ray scattering through the $\theta=90^{\circ}$ pitch angle in momentum space by wave-particle mirror interaction. By constructing a self-consistent model of cosmic ray propagation in the interstellar medium we were able to derive an expression for the particle diffusion coefficient and to estimate their residence time in the disk.

Scattering near the $\theta \sim 90^{\circ}$ pitch angle is produced by the particle mirror interaction with long wavelength waves generated by particles with $\theta \sim 0$ by the quasi-linear resonant interaction. In Sec. 2 we have shown how quasi-linear theory alone cannot account for particle crossing the $\mu=0$ point. We calculated the wave spectrum and the cosmic ray distribution function and we showed that the expression for the diffusion coefficient is a singular at $\mu=0$. We discussed how ion-cyclotron damping could further exacerbate the problem. In Sec. 3 we included the effect of mirroring and solved the diffusion 
equation numerically and we showed that the singularity is naturally removed by adiabatic wave-particle interaction. We then re-calculated the cosmic ray diffusion coefficient and showed that the effect of mirror interaction appears in the expression for $\mathcal{D}$ as the natural logarithm of the cutoff $\mu_{c}$. The critical cutoff is itself determined self-consistently from the model. We showed that cosmic ray are mainly confined in the hot regions of the interstellar medium and we calculated the particle residence time in the disk and found that our model predicts $t_{\text {life }} \approx 4.5$ Myrs, which is consistent with the observed particle residence time in the dense disk as deduced from the chemical composition. Finally, in Sec. 4 we derived the energy dependence of the diffusion coefficient to find that the result in the coronal regions, $\mathcal{D} \propto \gamma^{0.85}$, is consistent with the experimental evidence derived from the cosmic ray anisotropy which predicts a somewhat flatter power law. We extend the earlier work by (Cesarsky and Kulsrud 1981) by deriving the energy dependence of the particle drift velocity in the few $\mathrm{GeV}$ range.

\subsection{Open Problems and Future Work}

By resolving the $90^{\circ}$ problem we have shown that cosmic ray scattering through the $\theta=90^{\circ}$ point is efficient. Resolving this problem does not solve the problem of cosmic ray escape from the disk. This very important, and yet unresolved, problem is ultimately connected to the topology of the galactic magnetic field. Either the lines are permanently open to the extragalactic medium, or they are sporadically broken open by violent events in the galactic disc. Otherwise, because of their small gyration radius they could never escape contrary to their observed composition.

Parker (1992) proposed a possible explanation to the escape problem. The idea is that cosmic ray pressure could build up in the disk until it would drive magnetic bubbles near the edge of the disk. Cosmic rays would be partially trapped in the bubbles and ultimately 
they could escape together with part of the magnetic flux when the bubble finally detaches. The creation of magnetic bubbles would be helped by the explosion of supernovae close to the boundary of the disk or by the superposition of many SN's explosions (so called superbubbles). Ferrière $(1992,1993)$ studied the dynamics of magnetic bubbles expansion taking into account the effect of a distribution of SNs explosions. Very recently Rafikov and Kulsrud (2000) expanded this work and determined that the gravitational pull from the disk on the matter attached to the field lines would prevent significant amount of magnetic flux to leave the disk. Therefore, the cosmic ray escape problem is still very much an open question.

Finally, in this paper, we have not considered the important problem of the other possible sources of magnetic turbulence. In this paper galactic cosmic ray anisotropy is the only source of hydro-magnetic waves. An important instance in which energetic particle anisotropy is not the main source for the creation of a spectrum of waves occurs in interplanetary space (Dröge 1993). Energetic MeV particles originate from solar flares and other explosive events on the surface of the sun. These particles propagate outward from the sun and we detect them on earth. The propagation of these particles in the interplanetary medium is also regulated by their interaction (quasi-linear plus adiabatic) with hydro-magnetic waves (Völk 1975). However, this magnetic turbulence mainly originates at the sun. Its intensity is measured by satellites orbiting the earth. The analysis in this scenario is not qualitatively different from the one presented in this paper, the main difference being that the energy in the waves and the functional form of the wave spectrum is not determined self-consistently from balancing the damping rate with the growth rate. Rather, it is obtained from observation.

The result of an analysis of these results that ignores the 90 degree problem, is an order of magnitude discrepancy between the predicted spatial diffusion and the observed one. 
In this paper we did not elaborate on these important application, which is left for future research.

We wish to thank Roscoe White for many insightful discussions on the physics in the boundary layer region near $90^{\circ}$. This work was supported by DOE under contract No. DE-AC 02-76-CHO-3073 


\section{REFERENCES}

Achterberg, A. 1981 A\&A, 80,161.

Cesarsky, C.J. 1980, Rev. Astron. Astrophys, 8,189.

Cesarsky, C.J. 1987, Proc. 20th Int. Cosmic Rays Conf. (Moscow), 8,87.

Cowie, L.L., and Songaila, A., 1986 , An. Rev. Astron. Astrophys, 24,499.

Spitzer, L. 1978, Physical Processes in the Intersterllar Medium, J. Wiley, New York.

Dröge, W., 1993, Heliospheric Propagation of Cosmic Rays, 23rd International Cosmic Rays Conference - Calgary, Canada

Press et. al., 1992, Numerical recipes in C : the art of scientific computing, Cambridge [England]; New York: Cambridge University Press.

Ferrière, K., 1992, ApJ, 391,188.

Ferrière, K., 1993, ApJ, 409,248.

Foote, E.A., and Kulsrud, R.M., 1979, ApJ, 233,302.

Goldstein, M.L. 1976, ApJ, 204,900.

Goldstein, M.L. 1980, Geophys. Res., 25,3033.

Holman, G.D., and Ionson, J.A., and Scott J.S. 1979, ApJ, 228,576.

Kadomtsev 1965, Plasma Turbulence, London, New York, Academic Press.

Klimas, A.J., and Sandri, G. 1973, ApJ, 180,937.

Krall and Trivelpiece, 1990, Principles of Plasma Physics, McGraw-Hill, New York. 
Kulsrud, R.M. 1978, Astronomical Papers dedicated to B.Strømgren, pag. 317, Copenhagen Univ.Obs.

Kulsrud, R.M., and Cesarsky, C.J. 1971, ApJ, 8,189.

Kulsrud, R.M., and Pearce, W.P. 1969, ApJ, 156,445.

Parker, E.N. 1992, ApJ, 401,137.

Rafikov, R.R. and Kulsrud, R.M. 2000, MNRAS, 314,839.

Stix, T. 1992, Waves in Plasmas, A.I.P., New York.

Völk, H.J. 1973, Astrophys. Space Science, 25,471.

Völk, H.J., and Cesarsky, C.J. 1982, Z. Naturforsch,37a,809.

Völk, H.J. 1975, Reviews of Geophysics and Space Physics, 13,547.

Wentzel, D.G. 1969, ApJ, 156,303.

Cesarsky, C.J., and Kulsrud, R.M. 1981, Origin of Cosmic Rays, 251-252, Setti, G., Spada, G., Wolfendale, A.W. (eds.), IAU

Felice, G.M., Ph.D. Thesis, Princeton University, 2000

\section{A. Appendix A - Trapping and detrapping in non-linear Landau damping}

In order to determine if trapping of the thermal ions in the potential well of the beat wave of two interacting Alfven waves could set it, we first determine the trapping time 
and then compare it with the wave decorrelation time (i.e. the lifetime of the beat wave). Alfven waves propagating in a finite $\beta_{p}$ plasma have finite dispersion, where $\beta_{p}=8 \pi n_{i} T_{i} / B^{2}$ is the ratio of the background ion thermal pressure to the magnetic field pressure. The decorrelation time $t_{d e c}$ is (Stix 1992)

$$
t_{d e c}=\frac{1}{k\left(V_{g}-V_{p}\right)}
$$

where $V_{p}$ is the wave phase velocity and $V_{g}$ is the wave group velocity. In the laboratory frame, the large $\beta_{p}$ Alfven wave dispersion relation is (Foote and Kulsrud 1979).

$$
\left(\omega^{2}-k_{\|}^{2} V_{A}^{2}\right)^{2}=\frac{1}{2} \frac{\omega^{2} k_{\|}^{4} V_{i}^{4}}{\Omega_{o}^{2}}
$$

Treating the right hand side of the dispersion relation as a correction we obtain:

$$
\left(V_{g}-V_{p}\right) \approx \frac{1}{2 \sqrt{2}} \frac{k_{\|} V_{i}^{2}}{\Omega_{o}} .
$$

Therefore, the decorrelation time is:

$$
t_{d e c}=\frac{\sqrt{8}}{\Omega_{o}}\left(\frac{c}{V_{i}}\right)^{2} \mu^{2}
$$

where we made use of the resonant condition $k v \mu=\Omega_{0}$ to express $k$ in terms of $\mu$.

Let us now derive an expression for the trapping time. The force experienced by an ion in the potential well of the wave is Eq. (20), with $v_{\perp} \approx V_{i}$. The approximate bounce frequency of the ion as:

$$
\omega_{b} \simeq \sqrt{\frac{k_{\|} F}{m}}=\frac{v_{\perp}}{2} k_{\|}\left(\frac{\delta B}{B}\right)
$$


and the trapping time is then $t_{\text {trap }}=1 / \omega_{b}$ :

$$
t_{\text {trap }} \simeq \frac{2}{v_{\perp}} \frac{\mu c}{\Omega_{o}} \frac{1}{(\delta B / B)}
$$

The ratio $t_{\text {dec }} / t_{\text {trap }}$ is then:

$$
\frac{t_{d e c}}{t_{\text {trap }}}=\left(\frac{c}{V_{i}}\right) \frac{\mu}{\sqrt{2}}\left(\frac{\delta B}{B}\right)
$$

where $v_{\perp} \approx V_{i}$. On the other hand, particles will tend to get untrapped due to binary ion-ion collisions. The collision rate can be expressed in terms of the collision cross-section $\sigma$ as:

$$
t_{i i}^{90}=\frac{1}{n_{i}<\sigma v_{i}>}
$$

where $t_{i i}^{90}$ is the time it takes an ion to scatter a full 90 degrees in velocity space. In our case, the background Maxwellian distribution is distorted only slightly due to particle trapping. Indeed, to became untrapped, particles only need to scatter a small angle $\Delta \theta=\Delta v_{\|} / V_{i} \sim(\delta B / B)$. Therefore, the effective ion-ion scattering time is much shorter than the 90-degree scattering time.

$$
t_{i i}^{e f f}=t_{i i}^{90} \times(\Delta \theta)^{2}=\frac{(\delta B / B)^{2}}{n_{i}<\sigma v_{i}>} .
$$

The $90^{\circ}$ ion-ion cross section is $\sigma \simeq 10^{-12} / T_{i}^{2}(\mathrm{eV})$, and for a thermal distribution we have:

$$
t_{i i}^{e f f}=\frac{(\delta B / B)^{2}}{n_{i} \sigma V_{i}}=10^{12} \frac{T_{i}^{2}(e V)}{n_{i} V_{i}}\left(\frac{\delta B}{B}\right)^{2}
$$

or with $V_{i}=10^{6} T_{i}^{1 / 2}(\mathrm{eV})$ 


$$
t_{i i}^{e f f}=10^{6} \frac{T_{i}^{3 / 2}(\mathrm{eV})}{n_{i}\left(\mathrm{~cm}^{-3}\right)}\left(\frac{\delta B}{B}\right)^{2} \quad \mathrm{sec} .
$$

If collisions are fast enough to untrapped most of the particles, then saturation of non-linear

Landau damping will not take place. Therefore we are interested in the ratio $t_{i i}^{e f f} / t_{\text {trap }}$. In Table 1 we present some typical parameters of interest for the coronal region and estimate the various time scales, the decorrelation time, the trapping time, the ion-ion collision time and the non-linear Landau damping time as obtain from Eq. (13). It can be seen that in every case the trapping time is faster than the decorrelation time, $t_{\text {dec }} / t_{\text {trap }}>1$, but the effective collision time is faster than every other time scale, $t_{i i}^{e f f} / t_{\text {trap }}<1$. Therefore we argue that it is appropriate to use the unsaturated damping rate. Note that as $\mu$ decreases, the situation only gets better, as the amplitude of the wave decreases and the effective ion-ion collision time decreases as well. Moreover, the ratio of the decorrelation time to the trapping time, Eq. (A7), decreases, so thermal ions are less and less trapped.

\section{B. Appendix B - Parallel Propagation of the Waves}

Throughout the paper we have assumed that $k_{\perp} \ll k_{\|}$, so that the waves are nearly all parallel propagating. A more accurate expression for the growth rate, including its dependence on $k_{\perp}$ can be written

$$
\Gamma_{g}(k)=4 \pi^{2} e^{2}\left(\frac{V_{A}}{c}\right)^{2} \int d^{3} p v_{\perp}^{2} \frac{1}{V_{A} p} \frac{\partial F(p, \mu)}{\partial \mu} \mathcal{L}(x) \delta\left(k v_{z}-\Omega_{o}\right)
$$

The factor $\mathcal{L}$ represents the $k_{\perp}$ dependence. For linearly polarized shear Alfven waves $\mathcal{L}=J_{1}^{2}(x) / x^{2}$, while for linearly polarized magnetosonic waves $\mathcal{L}=J^{\prime 2}(x)$. However, for small $k_{\perp}$ the waves are circularly polarized and $\mathcal{L}$ can be taken as $\approx(1 / 4)\left(1-x^{2} / 4\right)$. We are interested in this limit. Thus, the growth rate can be written as 


$$
\Gamma=\Gamma_{0}\left(1-\frac{k_{\perp}^{2} \rho^{2}}{4}\right)
$$

where $\Gamma_{0}$ is the growth rate for $k_{\perp}=0$, The growth rate is a maximum at $k_{\perp}=0$, and we expect the waves to be concentrated there.

In this appendix we estimate the size of $k_{\perp}$ for which the wave energy is significant, and we show that it is very small compared to $k$.

If we start with only waves at $k_{\perp}=0$, we expect that $k_{\perp}$ should change from zero because of refraction in the inhomogeneous interstellar medium. If $B$ is uniform but the density $n_{i}(x)$ is nonuniform perpendicular to $B$ with scale length $L_{\perp}$, then $d k_{\perp} / d t=-\partial \omega / \partial x \approx \pm \omega / 2 L_{\perp}$. Let us assume $\dot{k_{\perp}}$ is positive. As $k_{\perp}$ increases, the linear growth rate decreases. Balancing the linear growth against the nonlinear damping, which is independent of $k_{\perp}$, one finds that when $x=k_{\perp} \rho$ exceeds some critical value $x_{c}$ the waves damp.

Thus, the waves of interest for cosmic rays will be thermally excited at $k_{\perp}=-k_{\perp c}$, grow for the time it takes them to reach $+k_{\perp c}$, by refraction, and then damp.

In terms of $x$, the net growth, that is the linear growth minus the nonlinear damping, is:

$$
\Delta \Gamma=\Gamma_{0}\left(1-x^{2} / 4\right)-\Gamma_{0}\left(1-x_{c}^{2} / 4\right)=\frac{1}{4} \Gamma_{0}\left(x_{c}^{2}-x^{2}\right)
$$

The net number of efoldings for the wave amplitude is

$$
D=\int \Delta \Gamma d t=\int_{-x_{c}}^{x_{c}} \Gamma_{0} \frac{d t}{d x} d x=\frac{1}{3} \frac{L_{\perp}}{\rho \omega} x_{c}^{3} \Gamma_{0}
$$

Substituting from Eq. (11) for $\Gamma_{o}$ we find: 


$$
x_{c}=\left(\frac{3 \rho}{L_{\perp} A}\right)^{1 / 3} D^{1 / 3} .
$$

$D$ is the number of required exponential growths from a thermal level to the required amplitude for pitch angle diffusion. It is quite large. $\delta B / B \approx 10^{-4}$ corresponds to a temperature of $\approx 10^{29}$ degrees $\mathrm{K} .\left(d^{3} k \approx\left(10^{-12}\right)^{3} \approx 10^{-36}\right.$ and $\delta B^{2} / 8 \pi \approx 10^{-19}$ ergs per $\mathrm{cm}^{3}$.) Thus, starting from $10^{4}$ degrees the energy amplifies by $10^{25}$ and the amplitude by $3 \times 10^{12}$ so $D \approx 28$. Taking $L_{\perp}=100 \mathrm{pc}, \rho / L_{\perp} \approx 10^{-8.5}$ and $A=10^{-5}$ we find

$$
k_{\perp c} / k \approx k_{\perp c} \rho=x_{c} \approx 0.3
$$

An additional process which further reduces $x_{c}$ is transit time damping, which is fairly fast and acts mostly on the magnetosonic mode. Since $x$ is fairly small there is a coupling between the two modes (by the Hall term in Ohm's law) and both waves suffer linear damping in addition to nonlinear damping. A more careful treatment of these processes is given in the thesis of the first author (Felice 2000). 
Table 1: Astrophysical values for $L_{z}=3 \mathrm{kpc}$ and $n_{c . r .}=1 E-10 \mathrm{~cm}^{-3}$

\begin{tabular}{ccccccccc}
\hline \hline$T_{i}(\mathrm{eV})$ & $n_{i}\left(\mathrm{~cm}^{-3}\right)$ & $B(\mu G)$ & $(\delta B / B)^{2}$ & $t_{\text {dec }}(\mathrm{sec})$ & $t_{\text {trap }}(\mathrm{sec})$ & $t_{i i}^{90}(\mathrm{sec})$ & $t_{i i}^{\text {eff }}(\mathrm{sec})$ & $t_{n . l .}(\mathrm{sec})$ \\
\hline 100 & $1.0 E-3$ & 3.0 & $5.0 E-6$ & $9.2 E 8$ & $9.5 E 7$ & $1.0 E 12$ & $5.1 E 6$ & $3.4 E 10$ \\
100 & $1.0 E-2$ & 3.0 & $2.8 E-6$ & $9.2 E 8$ & $1.2 E 8$ & $1.0 E 11$ & $2.8 E 5$ & $6.0 E 10$ \\
100 & $1.0 E-3$ & 1.0 & $1.5 E-5$ & $2.8 E 9$ & $1.6 E 8$ & $1.0 E 12$ & $1.5 E 7$ & $3.4 E 10$ \\
10 & $1.0 E-3$ & 3.0 & $8.9 E-6$ & $9.2 E 9$ & $2.2 E 8$ & $3.2 E 10$ & $2.9 E 5$ & $6.0 E 10$ \\
10 & $1.0 E-2$ & 3.0 & $5.0 E-5$ & $9.2 E 9$ & $3.0 E 8$ & $3.2 E 9$ & $1.6 E 4$ & $1.1 E 11$ \\
10 & $1.0 E-3$ & 1.0 & $2.7 E-5$ & $2.8 E 10$ & $3.9 E 8$ & $3.2 E 10$ & $8.8 E 5$ & $6.1 E 10$ \\
\hline
\end{tabular}


The Princeton Plasma Physics Laboratory is operated by Princeton University under contract with the U.S. Department of Energy.

\author{
Information Services \\ Princeton Plasma Physics Laboratory \\ P.O. Box 451 \\ Princeton, NJ 08543
}

Phone: 609-243-2750

Fax: 609-243-2751

e-mail: pppl_info@pppl.gov

Internet Address: http://www.pppl.gov 